\title{
EarthArXiv Cover Page
}

2020-12-11

Inference of thermodynamic state in the asthenosphere from anelastic properties, with applications to North American upper mantle

Christopher Havlin

University of Illinois at Urbana-Champaign, chavlin@illinois.edu

Benjamin Holtzman

Lamont-Doherty Earth Observatory, Columbia University, benh@ldeo.columbia.edu

Emily Hopper

Lamont-Doherty Earth Observatory, Columbia University

This manuscript is a non-peer reviewed pre-print. It has been submitted for publication in Physics of the Earth and Planetary Interiors and is currently in review. As such, the content of the present manuscript may change until the final version is published, at which point a DOI link will direct you to the final published version. 


\title{
- Inference of thermodynamic state in the asthenosphere 2 from anelastic properties, with applications to North American upper mantle

\author{
Christopher Havlin, Benjamin K. Holtzman*, Emily Hopper
}

\begin{abstract}
Inference of thermodynamic state and full-spectrum mechanical behavior of the lithosphere and asthenosphere is a central problem in geophysics, implicating our understanding of the convection patterns, transient responses and chemical composition of the planet. Anelasticity is responsible for significant relaxation of stress associated with seismic wave propagation in the asthenosphere, while irreversible transient creep may be important in the lithosphere. This paper focuses on the processes that may act at the time scales of seismic wave propagation, and current questions in the effort to determine the dependence of these effects on thermodynamic state. We introduce a free code library, the "Very Broadband Rheology calculator" (VBRc), designed to calculate frequency-dependent mechanical properties and easily compare different constitutive models favored by different laboratories. The methods operate only in the forward sense, starting with arrays of models of thermodynamic state, proceeding to arrays of mechanical properties. These calculations are incorporated into a Bayesian framework to infer variation in mantle thermodynamic state from Vs and Q, applied here to four locations in Western North America. The results demonstrate how well we can constrain the state, given the input models and the measurements. Results for sites in the Basin and Range, Colorado Plateau and interior craton east of the Rio Grande separate into distinct state variable ranges consistent with their tectonic environments.

*corresponding author
\end{abstract}




\section{Introduction}

The aim of this paper is to present an integrative framework for calculating the effects of anelasticity on seismic velocity and attenuation, and then apply a Bayesian inference framework to several sites in western North America. Inference of thermodynamic state of the upper mantle is central to understanding the mechanics of the lithosphere, the spatial variations in the degree of mechanical coupling between plate motions and convection patterns, and any questions of melting productivity and extraction physics. It is also critical for understanding surficial expressions of the mantle responses to large earthquakes, ice sheet melting, and other transient loads. Transient creep contributes to anelastic (recoverable, time dependent) deformation that affects a wide range of processes in the Earth including dissipation of seismic wave energy,expressed as "physical" velocity dispersion and attenuation of wave amplitudes. The magnitude of intrinsic attenuation depends on a range of state variables critical to our understanding of upper mantle dynamics including temperature, pressure, chemical composition, melt topology, and other microstructural properties such as grain size, subgrainsize and dislocation density. Furthermore, the sensitivity of seismic shear wave speed to anelasticity varies from that of attenuation and so the two measurements can be used in tandem to refine the ranges of temperature, melt fraction and grain size that can explain observations.

This paper introduces our free and open source code library, called the "Very Broadband Rheology calculator" or VBRc (Havlin et al., 2020). Although it has been used in previous publications (Bellis and Holtzman, 2014; Holtzman, 2016; Byrnes et al., 2019; Lau et al., 2020; Accardo et al., 2020; Hopper et al., 2020), here we describe its contents in conjunction with its public release. The software is written in MATLAB (2017) but is also functional in GNU Octave (Eaton et al., 2015). The core of the library calculates elastic, viscous and anelastic properties as a function of thermodynamic states for large and flexible ensembles of variables. The underlying idea is that there is enough accumulated understanding in the rock mechanics community to build a framework that predicts mechanical responses to any forcing at any thermodynamic state across all relevant frequencies. This understanding is far from complete, but the holes can be illuminated by having a framework for their calculation. The aim is to be able to self-consistently predict the mechanical behavior at any time scale from an inference at any other time scale (e.g., Cooper, 2002; Takei, 2013; Lau and Holtzman, 2019; Lau et al., 2020). The heart of the VBRc is to use the anelastic constitutive models to infer mechanical behavior at any time scale relevant to geophysics, from completely unrelaxed to completely relaxed. In this paper, we only focus on the inference of thermodynamic state from seismic measurements within the seismic band. The code structure is also designed to be used to develop new constitutive models from laboratory data, such that, by virtue of being a public code repository, new models can quickly be used by geophysicists to interpret their measurements.

As the calculation is an entirely forward calculation, it can easily be used in the context of 
a Bayesian inference approach to infer thermodynamic state over some representative volume of upper mantle. Because there is significant uncertainty in both the seismic measurements and the extrapolation of mechanical properties from the laboratory, the Bayesian inference approach is valuable for telling us how well we can actually constrain the thermodynamic state given the current state of knowledge and limitations of the measurements at hand.

In the following, we first provide a non-comprehensive overview of the current state of understanding of olivine rheology that forms the basis for the VBRc. Subsequently in Section 2 we provide a detailed description of the elastic, viscous and anelastic calculations currently in the VBRc, including reproduction of experimental mechanical data. We include several anelastic constitutive models, as agnostically as possible. In Section 3 we describe the measurable seismic properties predicted by the VBRc. Section 4 describes tradeoffs between temperature, melt fraction and grain size for the different anelastic methods at upper mantle conditions. Finally, in Section 5 we introduce a Bayesian framework which we then use to infer the likely ranges of temperature and melt fraction for four representative sites in the Western U.S. using four different anelastic scalings and three different prior models that explore the role of grain or subgrain size on inference of temperature and melt fraction.

In the analysis of results from the Bayesian inference (Sections 5.2.2 and 5.3), we show that for a given site, the majority of anelastic methods produce similar probability distributions, with the test sites (Basin and Range, Colorado Plateau, Eastern North American cratonic interior) generally separating into distinct locations in likely temperature-melt space. Additionally, we explore three treatments of grain or subgrain size through the application of different prior model probability distributions for grain or subgrain size and show that for a lengthscale closer to subgrain sizes, inferred temperatures are dramatically lower than inferred temperatures at a lengthscale closer to grain size.

\subsection{Complex Rheology or Complex Composition?}

It is possible and maybe even useful to describe a spectrum of efforts to infer the thermo-chemical state in the Earth's interior characterized by two end-members: Complex composition, simple rheology (CCsr) and Complex rheology, simple composition (CRsc) The former is a much more voluminous literature, stemming from the merging of mantle petrology (phase equilibria), seismology and geodynamics communities. The general methodology entails building hypothetical mantle compositions, calculating the equilibrium phase assemblages as functions of pressure and temperature, then mapping the weighted averages of density and elastic properties of over the phases to $V_{p}, V_{s}$. (Duffy and Anderson, 1989; Goes et al., 2000; Goes and van der Lee, 2002; Cammarano et al., 2003; Hacker et al., 2003; Lee, 2003; Stixrude and Lithgow-Bertelloni, 2005; Connolly, 2005; Schutt and Lesher, 2006; Cobden et al., 2008; Afonso et al., 2008; Cammarano et al., 2009; Khan et al., 2009, 2011). Adding some attenuation to account for physical dispersion 
is necessary, and is often described by a temperature-dependent function for Q, (e.g. Goes et al., 2000; Cobden et al., 2008).

On the other end, the Complex rheology, simple composition approaches explore the variability that can arise from the anelasticity primarily, and has emerged as our understanding of anelasticity has rapidly expanded (e.g. Behn et al., 2009; Dalton and Faul, 2010; Priestley and McKenzie, 2013; Plank and Forsyth, 2016; Hoggard et al., 2020; Richards et al., 2020). The methods are perhaps more diverse as the effort is younger. For example, Priestley and McKenzie (2013); Richards et al. (2020); Hoggard et al. (2020) utilize an inverse path to calibrate uncertain parameters in the Yamauchi and Takei (2016) anelastic model by fitting a canonical velocity model for the oceanic upper mantle and assuming a well-constrained thermal structure. The gap between the CCsr and CRsc approaches is quite large, in our opinion, and needs to be bridged, in spite of the numerous additional parameters and uncertainties. Here we focus on the complexrheology end-member, with a focus on anelasticity, quantifying the uncertainty that comes from several existing anelastic scaling and fitting models using forward calculations.

\subsection{Anelasticity: background and current questions}

Anelasticity occurs for small strain processes that access dissipative transient creep processes when elasticity enables strain to be recovered with a time lag. In linear anelasticity, the constitutive models are not directly dependent on the amplitude of the forcing, when the energy input is not large enough to modify the microstructure (e.g. Cooper, 2002). However, the scaling laws for the constitutive models are not generally linear in frequency, temperature, pressure, nor on microstructural state variables. In non-linear anelasticity, the anelastic response is a non-linear function of the stress or strain amplitude, because the stress alters the microstructure, such as the creation of dislocations. At present, the VBRc only includes linear anelasticity, but non-linear constitutive models will be added in the future.

Valuable review papers have condensed significant recent progress in experimental studies of attenuation in geologic materials and analogues, (e.g. Cooper, 2002; Jackson, 2007; Takei, 2013; Faul and Jackson, 2015; Takei, 2017). In this section, we first present a brief overview of the processes associated with linear and non-linear anelasticity at high temperature. In particular, we focus on effects of melt discovered in experimental studies. Until recently, one fundamental difficulty with interpreting velocity and attenuation variations in terms of melt content was that models of elastic and viscous properties were derived with very different geometric descriptions of the melt topology. Takei (2013) described the aim to have a continuous description of melt effects across elastic and viscous properties, spanned by the anelastic behavior. This aim requires the use of a single, consistent description of melt geometry, the "contiguity" for elastic (Takei, 1998, 2002) and viscous (Takei and Holtzman, 2009a) end-members. With various scalar parameterizations for elastic and viscous effects, cast in terms of the melt fraction, but consistent with 
contiguity formulations, the VBRc is implemented towards the aims described by Takei (2013).

\subsubsection{High-temperature Background}

General agreement has emerged that there exists a "high temperature background" (HTB) attenuation mechanism in olivine, governed by transient diffusion creep (e.g. Gribb and Cooper, 1998; Cooper, 2002; Jackson and Faul, 2010). The mechanism is the consequence of small displacement on approximately inviscid grain boundaries induced by a passing seismic wave, causing stress concentrations at grain edges and faces (Raj and Ashby, 1971; Raj, 1975; Morris and Jackson, 2009), as illustrated in Fig. 1a-d, also referred to as diffusion-assisted grain boundary sliding (e.g. Faul and Jackson, 2015). As illustrated, those stress concentrations are dissipated by local diffusion from grain faces under compression to adjacent faces under relative tension. The amount of dissipation depends on the frequency of the wave with a power law dependence, $Q \propto f^{n \approx 1 / 4-1 / 3}$, consistent with the empirical Andrade model (Andrade, 1910, 1962), demonstrated theoretically by (Raj, 1975; Gribb and Cooper, 1998; Morris and Jackson, 2009). The model also predicts that the response of any material undergoing this process can be scaled to other thermodynamic conditions by the Maxwell frequency, the ratio of an unrelaxed elastic modulus to a steady state viscosty, $f_{m}=M_{u} / \eta$. If operating in the HTB regime, the data should collapse to one "master curve" if the frequency of the experiment is normalized by $f_{m}$, as demonstrated by Cooper (2002) and McCarthy et al. (2011). The rate controlling property in this HTB process is the kinetics of the fastest diffusive pathway to carry matter away from the stress concentration, be it the grain boundary, subgrain structure, or melt structure. An important question, and a source of disagreement among different workers, is the appropriate length scale associated with the steady state viscosity $\eta$.

Although the grain boundary structure is illustrated in 1a-d, another important possibility is that transient diffusion creep on the subgrain structure, when such structure exists, dominates the HTB dissipation (e.g. Gribb and Cooper, 1998; Cooper, 2002). A strong piece of evidence for this process come from scaling data from experiments on multiple materials, conditions and machines, by McCarthy and Cooper (2016) (their Fig. 5), building on that of Cooper (2002); McCarthy et al. (2011). They show that data from attenuation experiments on olivine single crystals (Gueguen et al., 1989) collapses onto the master curve when normalized by the Maxwell frequency using the diffusion creep viscosity $\left(\eta_{d i f f}\right)$ for the estimated mean subgrain size instead of the grain size. (In the single crystal experiments by Gueguen et al. (1989), the crystal was deformed before the attenuation experiment to produce the dislocation structures.) In many fine grained samples used for attenuation studies, the grain size is smaller than what the subgrains would be at microstructural steady state at the average stress of the experiment, which is why this observation is subtle but important, with significant broader implications. If dislocation creep is an important process in the convecting upper mantle, as strongly supported by ubiquitous measured seismic anisotropy 
and observed crystal lattice preferred orientations and microstructures in xenoliths and ophiolites, it may be that the subgrain size is the appropriate lengthscale for estimating the HTB attenuation, with minor or significant additional effects coming from the grain boundary structure.

That said, the similitude (collapse of data onto the master curve by normalizing by the Maxwell time) may also be oversimplified. McCarthy et al. (2011) showed that most experimental data that collapse onto the master curve are at least two orders of magnitude below the normalized frequency of the seismic band, much closer to the Maxwell time of the various materials (borneol, olivine). Subsequent work on a machine designed to operate at higher normalized frequency for borneol-based materials (Takei et al., 2014) has shown that the master curve scaling breaks down closer to the scaled seismic band. While scaling by diffusion creep viscosity in the Maxwell time predicts a cubic grain size dependence to the reference time scale (not to the Q directly, as the slope of $Q(f)$ is a fractional power law), other studies produce and predict a smaller grain size dependence ( $m \approx 1$ ) to the reference time scale (Jackson et al., 2014; Faul and Jackson, 2015, and references therein), that is not consistent with the diffusion creep flow law. Thus, there are many open and fundamjackson2014elastically,ental questions and physics to be understood within the HTB concept.

Below, we discuss various potential influences of melt, water and second phases on the HTB, and then additional mechanisms that can elevate attenuation levels above the HTB.

\subsubsection{Direct effects of melt on the HTB}

A few studies have explored the effects of basaltic melt on attenuation in olivine rocks (e.g. Gribb and Cooper, 2000; Xu et al., 2004; Jackson et al., 2004; Faul et al., 2004), and in borneol systems (McCarthy and Takei, 2011; Yamauchi and Takei, 2016; Takei, 2017), all of which find significant effects of melt. The challenge is to identify the multiple possible effects of melt on attenuation mechanisms, including enhancing the HTB, elastically accommodated grain boundary sliding (eaGBS) and melt squirt, the latter discussed below. Jackson et al. (2004); Faul et al. (2004) found a significant effect of a small melt fraction on the measured attenuation, similar to their subsequent creep study (Faul and Jackson, 2007), discussed below. McCarthy and Takei (2011) demonstrated that crossing the solidus causes a large increase in the attenuation response of partially molten borneol + diphenylamine. Initially, this increase was associated with the effect of small melt fractions on the steady state diffusion creep (Takei and Holtzman, 2009a; Holtzman, 2016). However, subsequent discoveries in the borneol-based partially rock analogue system (Takei et al., 2014; Yamauchi and Takei, 2016; Takei, 2017) demonstrate that for a temperature, $T$, and solidus, $T_{s}$, the dramatic weakening attributed to melt begins at about $T / T_{s}=0.95$, or $95 \%$ of the melting temperature. This subsolidus weakening appears in the steady state viscosity as well as the attenuation measurements. It is referred to as premelting and is attributed to increased grain boundary diffusivity due to a highly local increase in disorder rather than a direct 
effect of a distinct melt phase (Takei, 2019), illustrated in Fig 1a.2. There is also good evidence of this process in ice (e.g. Rempel et al., 2001).

\subsubsection{Effects of phase boundaries}

Phase boundaries generally have lower viscosity (Zhao et al., 2019) and faster transport than grain boundaries (Cukjati et al., 2019), so can alter the behavior of the HTB. Sundberg and Cooper (2010) performed attenuation experiments on polyphase olivine-pyroxene samples, and showed that they tend to be more dissipative than otherwise similar olivine samples, which they attribute to the presence of phase boundaries. This inference points to an important bridge between the "simple composition, complex rheology" and "complex composition, simple rheology" approaches. The composition will not just affect the reference elastic modulus, density and the steady state viscosity, but also the anelastic response, in a way that may not reflect linear mixing of end-member behaviors. Therefore, we caution against merging the two approaches only through the elastic modulus, until a clearer empirical sense is gained for the effects of phase boundaries on steady state and transient creep. As discussed below, effects may emerge in only specific frequency bands, that if mis-accounted, could significantly alter ones interpretation of thermodynamic state.

\subsubsection{Direct effects of water on HTB mechanisms}

Dissolved water $\left(\mathrm{H}^{+}\right.$and $\mathrm{OH}^{-}$defect complexes) in nominally anhydrous minerals (NAMs) such as olivine and pyroxenes will increase diffusional kinetics in the crystal and possibly in grain boundaries (Hirth and Kohlstedt, 2003). Attenuation experiments with dissolved water are difficult to perform and interpret (e.g. Aizawa et al., 2008; Cline II et al., 2018), with the latter suggesting that water does not affect attenuation significantly, but the redox state does. It is difficult to understand how, if the steady-state diffusion creep rate is affected, the attenuation is not. One possibility suggested by Abers et al. (2014) is that dissolved water could enhance grain growth, counteracting the direct effect on diffusion kinetics. It is also important to note that because water lowers the solidus significantly, the interactions with melt should be considered but these are open questions that are beyond the scope of the present study.

A host of additional mechanisms beyond transient diffusion creep can absorb seismic energy, illustrated in Fig. 1c1-c4. In the following sections, we describe them briefly for completeness and a sense of the current scope of the VBRc. In the VBRc, we incorporate only the HTB at present, but secondary effects will be progressively added. In this paper, we focus on the effects of temperature, grain size, and melt on the HTB.

\subsubsection{Elastically-accommodated grain boundary sliding}

Zener (1941) developed the idea of elastically accommodated grain boundary sliding as a dissi- 
pative mechanism, with the grain boundary's viscosity giving rise to a energy loss with a narrow characteristic frequency. Transient diffusion creep invariably involves small-displacement sliding on grain boundaries (hence the term "diffusionally assisted GBS") (e.g. Raj, 1975; Cooper, 2002; Morris and Jackson, 2009; Faul and Jackson, 2015)). In discussions of HTB attenuation, when data exhibit only a $Q \propto f^{1 / 3}$ behavior, this sliding is assumed to be on effectively inviscid grain boundaries; the sliding dissipation is overwhelmed by the transient diffusion creep and is ignored. However, in certain conditions, significant portion of the displacement on the grain boundary can be accommodated by elastic distortion of the grains and recovered, driving sliding on the grain boundaries that can emerge above the HTB, and appear as dissipation peaks (Raj, 1975; Morris and Jackson, 2009), potentially in the seismic band. The frequency of these peaks is related to that grain boundary viscosity $\eta_{g b}$ as $\tau_{e a g b s}=\frac{\eta_{g b} d}{G \delta}$, where $d$ is the grain size, $G$ the shear modulus, and $\delta$ the thickness of the grain boundary. A decrease in $\eta_{g b}$ leads to an increase in the center frequency of the attenuation peak.

In some experimental data, peaks attributed to eaGBS emerge (e.g. Xu et al., 2004; Sundberg and Cooper, 2010; Jackson et al., 2006) but generally require some secondary effect present. (Sundberg and Cooper, 2010) added 40\% OPX to olivine and observed an increase in the HTB dissipation and the emergence of a clear peak (though only could sample one side of it). Jackson et al. (2006) argue that melt pockets significantly reduce the resistance to grain boundary sliding and so enable the emergence of the eaGBS peak and possibly melt squirt (Faul et al., 2004), discussed below. Karato (2012) hypothesized an effect of water on eaGBS that moves the peak into the seismic band and causes a strong apparent velocity contrast, but such effects have yet to be demonstrated in experiments. Yamauchi and Takei (2016) were able to resolve the full peak shape but attribute the peak to a solid state mechanism related to transient diffusion creep, not eaGBS (Takei, 2017). In short, there is much uncertainty in the scaling of frequency and amplitude of these secondary peaks associated with eaGBS.

\subsubsection{Dislocation damping mechanisms}

Dislocation damping is the process of attenuation by small-scale motions on an existing dislocation structure (Fig. 1c2) has been hypothesized (e.g. Minster and Anderson, 1980) and measured in experiments (e.g Farla et al., 2012; McCarthy and Cooper, 2016; Sasaki et al., 2019). These processes are closely related to the discussion above on the subgrain structure, as the mobile or free dislocation population is statistically related to the subgrain structure that could be undergoing elastically assisted (sub)grain boundary sliding (e.g. Cooper, 2002). Dislocation damping processes can straddle the boundary between linear and non-linear anelasticity. If small dislocation motions are reversible (but time-dependent) in the context of a passing seismic wave, and the stress amplitude of the seismic wave is not large enough to produce new dislocations, then the seismic wave has not changed the microstructure and the damping can be described by a dislo- 
cation density and/or subgrain structure that reflects the background stress level but not the stress amplitude of seismic waves. A non-linear process would occur if the seismic wave (or other loading process) created new dislocations beyond the pre-existing level and/or the degree of damping (distance traveled by kinks, for example) is stress amplitude-dependent. In experiments on ice, McCarthy and Cooper (2016) observed a grain size independence to the attenuation magnitude, implicating dislocations, but also a broad enhancement above the HTB but with a similar slope, similar to Farla et al. (2012). In contrast, in experiments on borneol, Sasaki et al. (2019) inferred a relatively narrow peak due to dislocations. Experiments with stable dislocation structures at appropriate high $\mathrm{T}$ conditions are quite difficult to do and much remains to be understood from the complex and multi-scale behavior of dislocations, as they may be important contributors to actual seismic attenuation in the mantle.

\subsubsection{Melt squirt and other mechanisms}

Melt squirt is an additional absorption mechanism in which the strains imposed on a partially molten rock by a passing seismic wave or other stress pulse drive melt flow over small distances and back during their passage. A range of possible behaviors definined by the boundary conditions on melt flow has been explored (e.g. Mavko and Nur, 1975; O'Connell and Budiansky, 1977; Schmeling, 1985) and more recently by Hammond and Humphreys (2000). The time scale depends on the melt viscosity and distribution of available length scales for melt flow. Melt squirt attenuation in meso-scale structures such as organized melt networks (Fig. 1c2-c4) has not yet been quantitatively modeled, to our knowledge.

Another additional possible mechanism involves different local thermal changes in phases, due to their different thermal expansion coefficients, driven by seismic strains (e.g. Budiansky et al., 1983; Chrysochoos, 2012). The frequency dependence of the dissipation emerges due to the relationship between the wave period and thermal exchange coefficients between adjacent phases or anisotropic phases. This mechanism has been only minimally explored.

\subsubsection{A note on self-consistency}

In the lab, a single experiment can measure the elastic, transient and steady state viscous properties. In practice, to calculate a full-spectrum mechanical response for rocks, an inherent source of complexity and uncertainty is the extrapolation from lab-to-earth conditions, and to the wide array of natural rock compositions and thermodynamic conditions that can only be minimally explored in the lab. Self-consistent calculation across the wide array of parameters can be achieved approximately, and we wish to point out here two aspects that emerged in the above discussions, namely (1) the relationship between microstructure and attenuation mechanisms and (2) the rock composition.

(1) While most of the laboratory experiments on attenuation have been performed on very fine 
grain samples, in the linear anelastic regime characterized by transient and steady state diffusion creep, it is likely that dislocation creep is at least an equal contributor to mantle deformation. If so, the microstructure characterized by subgrain and grain structures may significantly depart from that linear anelastic regime, and instead have a HTB response characterized by the subgrain size, and additional transient mechanisms related to dislocations themselves. Melt in this circumstance may change the balance of diffusion and dislocation dominated creep mechanisms as it affects the grain boundary structure, not the subgrain structure, to first order.

(2) While it is tempting to estimate effects of varying mantle composition by the simplest route: varying anharmonic elastic moduli and density as functions of composition and then superimposing anelastic behavior calculated for olivine rocks, the effects of phase boundaries will be ignored, which may be significantly incorrect. Composition effects need to be accounted for across the entire spectrum, from unrelaxed to steady state creep, but may cause an increase in attenuation that would not be captured by the end-member effects on the Maxwell time alone, though that is the place to start.

Clearly, there are many open questions. We do not try to include these effects or calculate any uncertainty from the constitutive models- doing so is beyond the scope of this paper. We also remain agnostic to the different fitting and scaling models employed here, but in future work intend to carry out comparisons of fitting models across laboratory data, towards convergence on the many questions above.

\section{Constitutive models in the VBRc}

Here, we describe the constitutive models for elasticity, viscosity and anelasticity that are currently included in the VBRc and implemented in this paper. At present they are limited to upper mantle applications, with particular focus on asthenosphere conditions. In general, the calculations proceed sequentially: elastic and viscous properties and calculated followed by anelastic calculations that inherit the elastic and viscous properties as needed. The following methods involve a large number of parameters and constants that are fit to expirimental values. Given that we are using values directly from the cited studies, we do not report the numerical values of each parameter here but note that the default values of all parameters in the VBRc can be easily loaded, viewed and adjusted at will by the user, as described in the online VBRc documentation.

\subsection{Elasticity}

The VBRc includes methods for calculating isotropic elastic properties accounting for anharmonic and poroelastic dependences on state variables. Of the physical properties calculated by the VBRc, the purely elastic properties are probably the best understood empirically and theoretically. However, as discussed in the Introduction, the compositional variations in the unrelaxed 
reference elastic moduli for all possible mantle phases are the basis for the large number of studies that focus on explaining velocity variations by thermal and compositional variations alone (e.g., Cammarano et al., 2003, 2009). As such, the VBRc focuses on understanding the complexity within anelastic methods and treats anharmonic and poroleastic effects in a simplified manner, as described below. One important note is that although the VBRc currently only calculates isotropic properties, it could be coupled to methods for adding anisotropic perturbations to the absolute velocity values.

\subsubsection{Anharmonicity}

Anharmonicity in the mineral physics context refers to deviations from harmonic oscillations of atoms in a lattice structure due to asymmetry in the attractive and repulsive forces among neighboring atoms. These asymmetries give rise to dependence of the elastic moduli on pressure, temperature and composition (e.g. Kumazawa and Anderson, 1969; Stixrude, 2007). At present, we treat anharmonicity as simply as possible using a linear scaling of a generic modulus $M$ from reference pressure $P_{R}$ and temperature $T_{R}$ :

$$
M_{u}(T, P)=M_{u 0}\left(T_{R}, P_{R}\right)+\left(T-T_{R}\right) \frac{\partial M}{\partial T}+\left(P-P_{R}\right) \frac{\partial M}{\partial P}
$$

The VBRc includes sets of anharmonic derivatives from Isaak (1992) and Cammarano et al. (2003) for Fo90 olivine and in this paper, we employ those of Isaak (1992): $d G / d T=-13.6$ $\mathrm{MPa}{ }^{\circ} \mathrm{K}$ and $d G / d P=1.8$ with a value of $G_{0}=80$ at standard temperature and pressure.

\subsubsection{Poroelasticity}

The effect of melt on the elastic properties of materials, the "poroelastic" effect arises from lowmodulus inclusions of melt or other fluid embedded in a matrix, and depends on the conditions of fluid mobility (e.g. O'Connell and Budiansky, 1977; Hammond and Humphreys, 2000). We account for the poroelastic effect of melt using the contiguity model of (Takei, 1998), implementing the parameterization of the isotropic solutions detailed in Appendix A of Takei (2002) that assumes drained conditions (i.e. constant fluid pressure). Parameterizations of more recent numerical approaches for calculating poroelastic effects (e.g. Hier-Majumder, 2008; Hier-Majumder and Drombosky, 2015) can be incorporated for comparison, but are not at present. To summarize, we can describe the above calculation path with this notation: $G_{0}\left(\{C\}_{c}\right) \rightarrow G\left(T, P, G_{0}\right) \rightarrow$ $G_{\text {poro }}(\phi, G)$.

\subsection{Steady-state viscosity}

The VBR calculator currently incorporates two sets of steady state flow laws for San Carlos olivine: Hirth and Kohlstedt (2003), incorporating diffusion, dislocation creep of dry or "wet" 
olivine, and Hansen et al. (2011) incorporating dry diffusion, dislocation and dislocation- accommodated grain boundary sliding (disGBS) creep. These mechanisms are assumed to act in kinetic parallel or mechanical series, so the total strain rate is then given by $\dot{\varepsilon}=\sum \dot{\varepsilon}_{i}$, where an individual mechanism's strain rate is given by

$$
\dot{\varepsilon}_{i}(\sigma, d, T)=C_{i}^{o} \sigma^{n_{i}} d^{-m_{i}} \exp \left(-\frac{Q_{i}+P V^{*}}{R T}\right)
$$

$i=1$ for diffusion creep, $i=2$ for dislocation creep and $i=3$ for GBS creep. $(\operatorname{sgn}(\sigma)=\operatorname{sgn}(\dot{\varepsilon})$ is implied.) $d$ is grain size, $Q_{i}$ is the thermal activation energy, $\sigma$ is the differential stress, and $V^{*}$ is the activation volume. Given the strain rate for each mechanism, the VBRc calculates the viscosity of a single mechanism, $\eta_{i}=\sigma / \dot{\varepsilon}_{i}$, as well as the total effective viscosity using the composite strain rate, $\boldsymbol{\eta}=\sigma / \dot{\boldsymbol{\varepsilon}}$. Parameter values for each method are taken directly from Hirth and Kohlstedt (2003) and Hansen et al. (2011) and can be easily adjusted.

Experiments on steady state creep of partially molten rock have long shown that the strain rate of partially molten rock exhibits an exponential dependence on melt fraction (e.g. Hirth and Kohlstedt, 1995a,b; Xu et al., 2004; Kohlstedt and Hansen, 2015). In terms of viscosity, this can be written:

$$
\eta(\phi)=\eta_{0} \exp (-\lambda \phi)
$$

where $\eta_{0}$ is the flow law for the subsolidus viscosity and $\lambda$ may vary for each deformation mechanism, as melt will affect them differently.

However, new questions on effects of melt emerged when Faul and Jackson (2007) found that sol-gel olivine became a factor of about 40 weaker when very small amounts of basaltic melt were added. Much of this difference could be due to the increase of point defect concentrations in the lattice due to equilibration with chemical components that are introduced with the added melt composition. However, Takei and Holtzman (2009a,b) developed a model for diffusion creep based on the contiguity as a state variable description of melt distribution developed by Takei (1998) for poroelasticity. This model predicted a very rapid weakening of up to a factor of 5 at the onset of formation of a connected network of melt tubules. This effect of very small melt fractions helped to address the very large discrepancy between the truly melt-free and meltbearing sol-gel olivine. Subsequently, McCarthy and Takei (2011) discovered a similar dramatic weakening across the solidus in the borneol-based rock analogue system.

Holtzman (2016) proposed this simple parameterization that fits the contiguity model results fairly well:

$$
\eta(\phi)=\eta_{0} \exp \left(-\left(\lambda \phi+x_{c} \operatorname{erf} \frac{\phi}{\phi_{\mathrm{c}}}\right)\right)
$$

where $\phi_{c}$ is the critical melt fraction and $x_{c}$ is the weakening amplitude across $\phi_{c}$. It behaves much like the scalar approximation of the contiguity model $\eta(\varphi)=A \varphi^{1 / 2} \eta_{0}$, where $A=1 / x_{c}$ 
and $\varphi=1-2.3 \phi^{1 / 2}$, but does not have the singularity at $\phi=0$ and can be easily turned off with $x_{c}=0$. Holtzman (2016) discussed the possibility that most San Carlos olivine samples contain this critical melt fraction of about $10^{-5}$.

In the VBRc, the small melt fraction factor, $\exp \left(x_{c} \operatorname{erf} \frac{\phi}{\phi_{c}}\right)$, is applied as a "correction" to the flow laws from nominally melt free to a truly melt-free viscosity (i.e. the empirical flow law parameters would contain that extra weakening). Rudge (2018) demonstrated theoretically that the direct effect on viscosity at the onset of connected network formation is a factor of about $x_{c}=$ 1.4 rather than 5. Thus, there is a convergence towards the idea that the dramatic weakening upon melting mostly reflects atomic-scale grain-boundary effects that emerge before the formation of nano-tubes of connected melt.

The default in the VBRc code is to leave this effect off (i.e. $x_{c}=0$ ) but we demonstrate the effect in Section 4.2 as an extra strengthening below the solidus, not extra weakening above. This parameterization is not applied to the pre-melting model, to prevent double accounting.

\subsection{Anelasticity}

In this section, we describe the approaches used in fitting and scaling anelastic models to experimental data and emphasize those implemented in the VBRc. In general, the approach is to fit an anelastic model to experimental data, which can then be used to scale to conditions in the Earth. The models are generally different definitions of the "relaxation spectrum" as a function of period, $X(t)$ (Takei, 2013):

$$
X_{i j k l}(\tau)=\Delta_{i j k l}^{G B} X_{i j k l}^{G B}(\tau)+\Delta_{i j k l}^{\text {disl }} X_{i j k l}^{\text {disl }}(\tau)+\Delta_{i j k l}^{m e l t} X_{i j k l}^{m e l t}(\tau)
$$

where $\Delta$ is the "relaxation strength" and $X(\tau)$ is the relaxation spectrum. The methods described below are all approximations of the full relaxation spectrum.

The VBRc currently implements four anelastic methods encompassing a range of models and scalings (actual method names used by the VBRc in parantheses): the Andrade pseudoperiod (andrade_psp) following Jackson and Faul (2010), the extended Burgers pseudoperiod scaling (eburgers_psp) following Jackson and Faul (2010), the empirical relaxation spectrum fitting with Maxwell scaling (xfit_mxw) following Takei and the empirical relaxation spectrum fitting with pre-melting scaling (xfit_premelt) following Takei. The user can choose a single method or multiple methods for comparison. In each case, the anelastic method chosen will inherit the relevant anharmonic or viscous calculations required for the method. Furthermore, any of the parameters can be individually adjusted which is useful for understanding the influence of each parameter or for conducting a new fitting exercise. Note that the VBRc scripts to reproduce figures referred to in the following subsections are available in the Projects/1_LabData directory of the VBRc repository. The data from the associated studies, however, are not included in the present release. 


\subsubsection{Andrade model with pseudoperiod scaling: andrade_psp}

The Andrade model (Andrade, 1910) takes the form of the exponential decay of transient creep, which subsequently became associated with grain boundary diffusion creep (Raj, 1975), with the following creep function:

$$
J(t)=J_{U}+\beta t^{n}+\frac{t}{\eta_{s s}} .
$$

where $\eta_{s s}$ is the steady state viscosity. Taking the Laplace transform of the creep function yields the storage and loss compliances, $J_{1}$ and $J_{2}$ as a function of angular frequency $\omega$ :

$$
\begin{aligned}
& J_{1}=J_{U}\left(1+\beta^{*} \Gamma(1+n) \omega^{-n} \cos (n \pi / 2)\right) \\
& J_{2}=J_{U}\left(\beta^{*} \Gamma(1+n) \omega^{-n} \sin (n \pi / 2)+\frac{1}{\omega \tau_{M}}\right)
\end{aligned}
$$

where $\Gamma$ is the gamma function, $\Gamma(n)=(n-1) !, J_{U}$ is the unrelaxed compliance, $\tau_{M}=\eta_{s s} J_{u}$ is the Maxwell time and $\beta^{*}=\beta / J_{U}$.

Following Jackson and Faul (2010), the "pseudoperiod master variable" approach for scaling from laboratory to earth conditions substitudes a master variable $X_{a}$ for the period in the angular frequency, $\omega=2 \pi / \tau=2 \pi / X_{a}$. The master variable $X_{a}$ is a function of the state variables measured from a reference state:

$$
X_{a}(T, P, d)=\tau_{0}\left(\frac{d}{d_{R}}\right)^{-m} \exp \left[\left(\frac{-E}{R}\right)\left(\frac{1}{T}-\frac{1}{T_{R}}\right)\right] \exp \left[-\left(\frac{V^{*}}{R}\right)\left(\frac{P}{T}-\frac{P_{R}}{T_{R}}\right)\right]
$$

where $\tau_{0}$ is the period of the oscillation.The VBRc implementation adds a dependence on melt fraction using the diffusion creep values for melt weakening from section 2.2:

$$
X_{a}(T, P, d, \phi)=X_{a}(T, P, d) \exp \left(\lambda \phi+x_{c} \frac{\phi}{\phi_{c}}\right)
$$

where the small-melt effect is off by default. The values of the free parameters $\beta *, n, E, V$ and $\tau_{M}$ are taken from the fit in table 1 of Jackson and Faul (2010). Fig. 2 shows the anelastic dependent modulus and attenuation vs. period with curves calculated using the VBRc and data from figure 1 of Jackson and Faul (2010).

At present, the only Andrade fitting parameters included in the VBRc are those of Jackson and Faul (2010). We intend for future updates to the VBRc to include additional fitting and scaling parameters based on the experimental work of Gribb and Cooper (1998), Cooper (2002) and Sundberg and Cooper (2010). Gribb and Cooper (1998) and Cooper (2002) present an Andrade model scaled by the Maxwell relaxation time for diffusion creep and Sundberg and Cooper (2010) developed a composite anelastic model comprised of an Andrade HTB and a wide Debye peak for a single relaxation time-scale process such as elastically accommodated GBS. 


\subsubsection{Extended Burgers model, pseudoperiod scaling: eburgers_psp}

The extended Burgers model is a phenomenological model of linear viscoelasticity that allows for the superposition of multiple relaxation mechanisms. The creep function $J(t)$ for the extended Burgers model is

$$
J(t)=J_{U}\left[1+\Delta \int_{\tau_{L}}^{\tau^{H}} D(\tau)\left(1-\exp \left(\frac{-t}{\tau}\right)\right) d \tau+\frac{t}{\tau_{M}}\right],
$$

where $D(\tau)$ is the distribution of relaxation times of the series of Kelvin elements and $\Delta$ is the "relaxation strength". The three relaxation times $\tau_{H}, \tau_{L}$ and $\tau_{M}$ each have a viscosity associated with them; $\tau_{M}$ is the Maxwell relaxation time (or period), which depends on the steady state viscosity, while the other two, (presumably) depend on viscosities that are determined by processes other than the steady state process.

Multiple relaxation mechanisms may be superimposed with different relaxation strengths. The distributions for the high temperature background attenuation of strength $\Delta_{B}$ and a dissipation peak with relaxation strength $\Delta_{P}$ are given by

$$
\begin{aligned}
D_{B}(\tau) & =\frac{\alpha \tau^{\alpha-1}}{\tau_{H}^{\alpha}-\tau_{L}^{\alpha}},\left[\tau_{L}<\tau<\tau_{H}\right] \\
D_{P}(\tau) & =\frac{1}{\tau \sigma_{p} \sqrt{2 \pi}} \exp \left(-\frac{1}{2}\left(\frac{\ln \frac{\tau}{\tau_{P}}}{\sigma_{p}}\right)^{2}\right) .
\end{aligned}
$$

where $0<\alpha<1$ when the relaxation time is between the high and low limits, $\tau_{L}<\tau<$ $\tau_{H}$ and the dissipation peak is a Gaussian distribution described by $\tau_{p}$ and $\sigma_{p}$. Including these two distributions in the creep function and transforming to the frequency domain results in the following storage and loss complicances:

$$
\begin{aligned}
& J_{1}=J_{U}\left[1+\Delta_{B} \int_{\tau_{L}}^{\tau_{H}} \frac{D_{B}(\tau) d \tau}{1+\omega^{2} \tau^{2}}+\Delta_{P} \int_{\tau_{L}}^{\tau_{H}} \frac{D_{p}(\tau) d \tau}{1+\omega^{2} \tau^{2}}\right] \\
& J_{2}=J_{U}\left[\omega \Delta_{B} \int_{\tau_{L}}^{\tau_{H}} \frac{\tau D_{B}(\tau) d \tau}{1+\omega^{2} \tau^{2}}+\omega \Delta_{P} \int_{\tau_{L}}^{\tau_{H}} \frac{\tau D_{P}(\tau) d \tau}{1+\omega^{2} \tau^{2}}+\frac{1}{\omega \tau_{M}}\right]
\end{aligned}
$$

Scaling to other conditions is achieved through scaling the Maxwell time, $\tau_{M}$, lower and upper integration limits $\tau_{L}$ and $\tau_{H}$, and dissipation peak time $\tau_{P}$ from reference values given activation energy $E$ and activation volume $V$ :

$$
\tau_{i}\left(T, P, d,\left[\phi, X_{H_{2} O}\right]\right)=\tau_{i R}\left(\frac{d}{d_{R}}\right)^{m} \exp \left[\left(\frac{E}{R}\right)\left(\frac{1}{T}-\frac{1}{T_{R}}\right)\right] \exp \left[\left(\frac{V^{*}}{R}\right)\left(\frac{P}{T}-\frac{P_{R}}{T_{R}}\right)\right]
$$


with $i=L, H, P, M$, grain size $d$, tempeature $T$, pressure $P$.

The VBRc includes four sets of fitting parameters that define the free parameters $\Delta_{B}, \alpha, \tau_{M} R$, $\tau_{L} R, \tau_{H} R, \Delta_{P}, \sigma_{p} \tau_{P} R, E$ and $V$. The sets of parameters are fits with the background only and the background plus peak for the single sample 6585 (table 1 of Jackson and Faul (2010)), the background only fit of the nominally melt-free specimens (table 2 of Jackson and Faul (2010)) and the best fitting background plus peak fit fo the nominally melt-free specimens (table 2 of Jackson and Faul (2010)).

The default behavior of the VBRc is to use the multi-sample background only fit, but all the fitting sets are stored so they can be easily toggled. The primary purpose of including the single sample fits is for benchmarking purposes. In figure 2, we reproduce figure 1 of Jackson and Faul (2010), showing the modulus and attenuation calculated by the VBRc for the extended Burgers pseudoperiod scaling both with and without the dissipation peak using the single sample fits for sample 6585. The remainder of this study uses the multi-sample fits.

\subsubsection{Chi-fit, Maxwell frequency scaling: xfit $m x w$}

An alternative approach to using a phenomenological model such as the Andrade or extended Burgers model is to use an empirical fit for the relaxation function itself (McCarthy et al., 2011; Yamauchi and Takei, 2016). Although the subsequent work of (Yamauchi and Takei, 2016) utilizes an improved machine with higher frequency range and a more precise fit to the data, we include the previous scaling model for its simplicity. In this case, $J_{1}$ and $J_{2}$ can be written (McCarthy et al., 2011):

$$
\begin{aligned}
& J_{1}(\omega, T, P, g)=J_{u}(T, P)\left[1+\int_{\tau=0}^{\tau=\text { inf }} X(\tau) \frac{1}{1+(\omega \tau)^{2}} \frac{d \tau}{\tau}\right], \\
& J_{2}(\omega, T, P, g)=J_{u}(T, P)\left[1+\int_{\tau=0}^{\tau=\text { inf }} X(\tau) \frac{\omega \tau}{1+(\omega \tau)^{2}} \frac{d \tau}{\tau}\right]+\frac{1}{\omega \eta},
\end{aligned}
$$

and the relaxation spectrum is empirical is given as a piecewise function that depends on the Maxwell-normalized period, $\tau^{\prime}$ :

$$
X(\tau)= \begin{cases}\beta_{1}\left(\tau^{\prime}\right)^{\alpha_{1}}, & \text { if } \tau^{\prime} \geq 10^{-11}, \\ \beta_{2}\left(\tau^{\prime}\right)^{\alpha_{2}}, & \text { if } \tau^{\prime}<10^{-11},\end{cases}
$$

where $\tau^{\prime}=\tau f_{M}$, with the Maxwell frequency $f_{M}$ given by

$$
f_{M}=\frac{1}{\tau_{M}(T, g, c, \phi)}=\frac{E_{u}(T, P, \phi)}{\eta_{\text {diff }}(T, g, c, \phi)},
$$

where $E_{u}$ is the unrelaxed modulus and $\eta_{d i f f}$ is the steady state diffusion creep viscosity of the material. 
The VBRc implements the two fits provided by McCarthy et al. (2011). In Fig. 3a and $3 \mathrm{~b}$, we plot the normalized relaxation spectrum and ratio of $J_{1} / J_{2}$ against Maxwell-normalized frequency, $f_{M}$, following Fig. 14 and 15 of McCarthy et al. (2011). Though the first fit (dashed curve) provides a better fit to $J_{1} / J_{2}$ as noted by McCarthy et al. (2011), the default behavior of VBRc is to use the second fit, which produces a relaxation spectrum curve that passes through the range for PREM.

\subsubsection{Chi-fit, Temperature-dependent (pre-melting) scaling: xfit_premelt}

The premelting model of Yamauchi and Takei (2016) ascribes the dramatic reduction in $\eta$ and $Q$ near the melting point to a change in the physical state and structure of grain boundaries at sub-solidus temperatures, prior to the formation of what would actually be called a melt phase. The resulting changes in mechanical properties should be continuous starting at about $T_{n}>$ 0.95 , where $T_{n}$ is the homologous, or solidus-normalized, temperature, $T_{n}=T / T_{\mathrm{s}}$ for a solidus temperature, $T_{\mathrm{s}}$.

The relaxation spectrum used by Yamauchi and Takei (2016) includes a background spectrum and a dissipation peak that depends on the homologous temperature, both of which are functions of the Maxwell-normalized timescale $\tau_{n}=\tau / \tau_{M}$ :

$$
X(\tau)=A_{B} \tau_{n}^{\alpha}+A_{p} \exp \left(-\frac{\ln \left(\tau_{n} / \tau_{n}^{P}\right)^{2}}{2 \sigma_{p}}\right),
$$

where $A_{p}$ and $\sigma_{p}$ are both piecewise functions of $T_{n}$ :

$$
A_{p}\left(T_{n}\right)= \begin{cases}0.01 & \text { if } T_{n}<0.91 \\ 0.01+0.4\left(T_{n}-0.91\right) & \text { if } 0.91 \leq T_{n}<0.96 \\ 0.03 & \text { if } 0.96 \leq T_{n}<1 \\ 0.03+\beta(\phi) & \text { if } 0.96 \leq T_{n}<1\end{cases}
$$

and

$$
\sigma_{p}\left(T_{n}\right)= \begin{cases}4 & \text { if } T_{n}<0.92 \\ 4+37.5\left(T_{n}-0.92\right) & \text { if } 0.92 \leq T_{n}<1 \\ 7 & \text { if } 0.96 \leq T_{n}<1 .\end{cases}
$$

The above relaxation spectrum results in the following relationships for $J_{1}$ and $J_{2}$ : 


$$
\begin{aligned}
& J_{1}\left(\tau_{n}\right)=J_{u}(T, P)\left[1+\frac{A_{B}\left(\tau_{n}\right)^{\alpha}}{\alpha}+\frac{\sqrt{2 \pi}}{2} A_{p} \sigma_{p}\left(1-\operatorname{erf}\left(\frac{\ln \left(\tau_{n}^{P} / \tau_{n}\right)}{\sigma_{p} \sqrt{2}}\right)\right)\right] \\
& J_{2}\left(\tau_{n}\right)=J_{u}(T, P) \frac{\pi}{2}\left[A_{B}\left(\tau_{n}\right)^{\alpha}+A_{p} \exp \left(-\frac{\ln \left(\tau_{n}^{P} / \tau_{n}\right)}{\sigma_{p} \sqrt{2}}\right)\right]+J_{u}(T, P) \tau_{n}
\end{aligned}
$$

Constant parameters include: $A_{B}, \alpha$ and $\tau_{n}^{P}$.

The steady state Maxwell time is given by $\tau_{M}=\eta / G_{U}(T, P)$ where $\eta$ is the steady state diffusion creep viscosity. Yamauchi and Takei (2016) introduce a scaling for the viscosity that includes a dependence on $T_{n}$ :

$$
\eta=\eta(T, P, d) A_{\eta}\left(T_{n}\right)
$$

where $\eta(T, P, d)$ is the viscosity at the current thermodynamic state (temperature, pressure, grain size)neglecting any pre-melt and direct melt effects and $A_{\eta}\left(T_{n}\right)$ has the form

$$
A_{\eta}\left(T_{n}\right)= \begin{cases}1 & \text { if } T_{n}<T_{n}^{\eta} \\ \exp \left(-\frac{T_{n}-T_{n}^{\eta}}{T_{n}-T_{n} T_{n}^{\eta}} \ln \gamma\right) & \text { if } T_{n}^{\eta} \leq T_{n}<1 \\ \gamma^{-1} \exp (-\lambda \phi) & \text { if } T_{n} \geq 1\end{cases}
$$

where $\gamma$ and $T_{n}^{\eta}$ are fitting constants and $\lambda$ is the steady state exponential melt dependence. Yamauchi and Takei (2016) write $\eta\left(T, P, d, \sigma_{d}\right)$ in terms of a reference state,

$$
\eta\left(T, P, d, \sigma_{d}\right)=\eta_{r}\left(\frac{d}{d_{r}}\right)^{m} \exp \left[\frac{H}{R}\left(\frac{1}{T}-\frac{1}{T_{r}}\right)\right] \exp \left[\frac{V}{R}\left(\frac{P}{T}-\frac{P_{r}}{T_{r}}\right)\right],
$$

with activation volume $V$, activation energy $H$, grain size exponent $m$, reference grain size $d_{r}$, reference temperature $T_{r}$, reference pressure $P_{r}$ and gas constant $R$. Any stress dependence is captured in the reference viscosity, $\eta_{r}$. To apply this relationship to borneol or olivine, $\eta\left(T, P, d, \sigma_{d}\right)$ is calculated with appropriate constants for either composition and $A_{\eta}\left(T_{n}\right)$ is the same for both.

The VBRc uses equation 25 with the values of $\eta_{r}, V$ and $H$ calculated by Yamauchi and Takei (2016) by fitting their anelastic model to the shear wave velocity of the Pacific upper mantle for the pre-melting anelastic scaling by default. But it also includes the option to use a laboratoryderived diffusion creep flow law to calculate $\eta(T, P, d)$. Figure $3 \mathrm{c}$ and $3 \mathrm{~d}$ shows a reproduction of the fit of borneol sample 41 (figure 10 and Table 4) of Yamauchi and Takei (2016) calculated using the VBRc.

\section{Measured properties}

The primary measured properties that are calculated by the VBRc at present are isotropic shear wave velocity $V_{s}$ and intrinsic attenuation $Q^{-1}$, 


$$
\begin{aligned}
V_{S} & =\sqrt{\frac{G}{\rho}} \\
Q^{-1} & =\frac{J_{2}}{J_{1}}
\end{aligned}
$$

Note that in the remainder below, we frequently refer to the quality factor, $Q$, the inverse of attenuation in lieu of $Q^{-1}$ as $Q$ varies with state variables in the same sense as $V_{s}$ : e.g., an increase in temperature decreases both $Q$ and $V_{s}$.

Measured properties as described here are generally derived from direct measurements of surface deformation or displacement of the Earth's surface, by seismic or geodetic methods. These measurements are related to combinations of the laboratory-derived mechanical properties defined above, some more direct than others, but all open to uncertainties coming from the lab, the forward calculation and the measurement in the Earth and derivation. Measurement of intrinsic attenuation in particular is a difficult prospect as it is derived from the observation of amplitude as a function of frequency, which is influenced by any combination of scattering, intrinsic attenuation and larger scale wave propagation effects such as focusing/defocusing (e.g., Zhou, 2009).

\section{Extrapolations to mantle conditions}

In this section, we compare predicted values of $V_{s}$ and $Q$ for different anelastic methods in the VBRc at mantle conditions. We discuss tradeoffs in choice of method as well as state variables and describe the generation of the look-up table used in the subsequent Bayesian Inference in section 5 .

\subsection{Melt effects: the importance of poro-elasticity}

The presence of a melt phase influences shear modulus, wavespeed and attenuation through both poro-elastic and anelastic effects. To demonstrate the importance of the poroelastic contribution to the measured velocity, we compare two paths in Fig. 4 without and with the poro-elastic effect. In the first, the anharmonic (unrelaxed) modulus contains no poro-elastic effect, so is not propagated forward into the anelastic calculation:

$$
\left(G_{\text {anh }}\left(T, P,\{C\}_{c}\right) ; \eta_{\text {diff }}\left(\phi, T, P, d,\{C\}_{c}\right)\right) \rightarrow J_{1}, J_{2}
$$

with the curly brackets indicating what properties are held constant. In the second case, the anharmonic (unrelaxed) modulus contains the poro-elastic effect:

$$
\left(G_{\text {anh }}\left(T, P,\{C\}_{c}\right) \rightarrow G_{\text {anh-poro }}\left(T, P, \phi,\{C\}_{c}\right) ; \eta_{\text {diff }}\left(\phi, T, P, g,\{C\}_{c}\right)\right) \rightarrow J_{1}, J_{2}
$$


The different anelastic models are influenced by melt fraction in different ways, as described in Section 2.3 and summarized in Table 1.

From Fig. 4, it is clear that the poro-elastic effect must be included when interpreting observed measurements. Fig. 4a and Fig. 4b show the final modulus and shear velocity, respectively, for the different anelastic methods when the poro-elastic effect of anharmonic modulus is included (solid curves) and is not included (dashed curves). Though each anelastic method depends on melt fraction differently in terms of the transient and steady state viscous relationships used, these curves indicate that once there is melt, the poro-elastic effect dominates the melt-dependence. This is true, however, only when there is melt.

\subsection{Near-solidus melt effects}

At very small melt fractions close to the solidus, the differences in the treatment of melt and melting by each anelastic methods result in important differences in predicted $V_{s}$ and $Q$. We compare two cases: in the first we fix the temperature at $1 \%$ above the solidus and vary melt fraction while in the second we calculate melt fraction as a function of temperature. The resulting properties (modulus $M$, shear velocity $V_{s}$ and quality factor $Q$ ) are calculated with and without the small melt effect described in Section 2.2 for all anelastic methods, except in the case of the pre-melting scaling which incorporates the near-solidus behavior into the pre-melting term rather than a small-melt effect (though we will show that the effects are similar). In Fig. 5, calcuations are done at $0.02 \mathrm{~Hz}, 1 \mathrm{~cm}$ grain size and $2 \mathrm{GPa}$, using the parametrization of Katz (2003) to calculate a dry solidus.

In the case of fixed temperature, Fig. 5a-d, the small-melt effect results in a strong drop in all parameters at the critical melt fraction, $\phi_{c}=10^{-5}$ for the pseudoperiod and Maxwell scalings. The effect on $M$ and $V_{s}$ is stronger for the pseudoperiod scalings compared to the Maxwell scalings while the effect on $Q$ is stronger for the Maxwell scaling. While the smallmelt effect adjusts the steady state Maxwell time in the same way in all the methods, there are additional influences that differ in each: the master variable $X_{a}$ in the Andrade pseudoperiod scaling and the integration limits $\tau_{L}$ and $\tau_{H}$ of the extendend Burgers pseudoperiod method are both modified by the small-melt effect, resulting in different responses in the final $M, V_{s}$ and $Q$. Fig $5 \mathrm{~d}$ demonstrates the sensitivity of steady state viscosity on the small-melt effect, showing that the drop in diffusion creep viscosity (light green curve) is larger than the drop in total viscosity (dark green curve). Note that the pre-melting scaling exhibits no dependence due to reasons discussed above and that the dependence on melt fraction above about $\phi=10^{-3}$ reflects the poroelastic dependence discussed in the previous section.

To calculate $\phi(T)$, we use a simple equilibrium batch melting calculation following Katz (2003) in which $\phi$ is given by the thermodynamic melt fraction, $\phi=F=\left(\left(T-T_{s}\right) /\left(T_{l}-T_{s}\right)\right)^{1} .5$ where $T_{s}$ and $T_{l}$ are the solidus and liquidus, respectively. Though melt in the mantle is buoyant 
and will segregate from the solid matrix, this formulation provides a simple, illustrative method for comparing the pre-melting scaling to the pseudoperiod and Maxwell normalization. Figure 5e plots $\phi T$, with dashed lines marking where $\phi(T)=\phi_{c}$ and $T=T_{s}$. The grey curve is a case in which we increase the solidus, discussed below.

By coupling $\phi$ to $T$, we can more directly compare all the anelastic methods. The behavior of the pseudoperiod and Maxwell scaling are similar to the fixed temperature case: $M, V_{s}$ and $Q$ decrease gradually as $T$ approaches $T_{s}$, solely from the temperature dependence of the scalings. At the solidus, the small-melt effect causes an effectively instantaneous drop in parameter values and the curvature a few degrees above the solidus (when $\phi$ reaches about $10^{-3}$ ), the poro-elastic effect dominates. The pre-melting scaling, however, decreases as it approaches the solidus. Interestingly, it effectively spans the other methods: at lower $T$, it is similar to the Maxwell scaling but as $T$ approaches the solidus, it approaches $M, V_{s}$ and $Q$ of the pseudoperiod scaling. If we increase the solidus temperature by $15^{\circ} \mathrm{C}$, the pre-melting scaling shifts as shown by the gray curves in figures $5 \mathrm{e}-\mathrm{f}$. This small change causes the pre-melting curve to match $M$ and $V_{s}$ of the Maxwell scaling quite well below and above the solidus, while near the solidus the pre-melting scaling smooths the transition from below-solidus to above-solidus behavior.

\subsection{Tradeoffs in grain size and temperature}

Similar to the influence of melt, the length scale for transient diffusion creep $d$, be it grain size or subgrain size, influences the anelastic methods in different ways. While the grain size exponents differ in the different methods ( $m=1.2$ in the ${ }^{*}$ PsP methods and $m=3$ in the Xfit* methods), the propagation of viscous terms into the relaxation spectrum and eventually $J_{1}$ and $J_{2}$ modulate the final dependence of $V_{s}$ and $Q$ on grain size.

To better understand the grain size dependence of the various methods, we pick two temperatures above and below the solidus from Fig. 5e-h and vary the grain size from $1 \mathrm{~mm}$ to $3 \mathrm{~cm}$. Fig. 6 shows the resulting $V_{s}$ and $Q$ for the four anelastic methods at temperatures of $1300^{\circ} \mathrm{C}$ (solid curves) and $1350^{\circ} \mathrm{C}$ (dashed curves). In the case of $T=1300^{\circ} \mathrm{C}$, the pre-melting method again spans the pseudoperiod and Maxwell scaling methods at low temperature: at smaller grain sizes, the pre-melting method is closer to the pseudoperiod methods while at larger grain sizes, the pre-melting method matches the Maxwell scaling fairly well. At higher temperatures $\left(1350^{\circ} \mathrm{C}\right.$, dashed curves), however, this pattern breaks down and the pre-melting scaling calculates signifcantly lower $V_{s}$ at small grain sizes. This difference highlights that while decreasing grain size and increasing melt both decrease $V_{s}$ and $Q$, they are not equivalent.

\subsection{Generation of a 3D Look-up tables for $\phi, d, T$}

Given the above description of melt and grain size dependencies for the different anelastic methods, we can move forward with using the VBRc to interpret observed values. In the following 


\begin{tabular}{|l|l|l|l|}
\hline state variables: & elastic: & viscous: & anelastic: \\
& $S_{e l}=\left[T, P,\{C\}_{c}\right]$ & $S_{v}=\left[T, P, \phi, d,\{C\}_{c}\right]$ & $S_{a n}=\left[T, P, \phi, d,\{C\}_{c} ; f\right]$ \\
\hline & $M_{0}(\{C\})$ & $\eta_{\text {diff. }}\left(S_{v}\right)$ & $\left.J^{*}\left(S_{a n} ; G_{\text {poro }}\right)\right|_{\text {and-PsP }}$ \\
$S_{i}=$ & $M_{a n h}\left(S_{e l} ; M_{0}\right)$ & $\eta_{\text {disl. }}\left(S_{v}, \sigma\right)$ & $\left.J^{*}\left(S_{a n} ; G_{\text {poro }}\right)\right|_{\mathrm{eB}-\mathrm{PsP}}$ \\
{$\left[T, P, \phi, d, \sigma,\{X\}_{c}\right]$} & $M_{\text {poro }}\left(\phi ; M_{a n h}\right)$ & $\eta_{g b s}\left(S_{v}, \sigma\right)$ & $\left.J^{*}\left(S_{a n} ; \tau_{M x w}\right)\right|_{\mathrm{Xfit}-\mathrm{mxw}}$ \\
& & & $\left.J^{*}\left(S_{a n} ; \tau_{M x w}\right)\right|_{\text {Xfit-premelt }}$ \\
\hline
\end{tabular}

Table 1: Flowchart for a generic example VBR configuration. First columns shows all state variables considered, with those in $\{-\}_{c}$ being held constant. In elasticity, the reference generic modulus $M_{0}$ (be it shear, $G$ or bulk, $K$ ) is a function of composition. The anharmonic value is then passed into the poro-elastic value calculation. In steady state viscosity, stress $\sigma$ and grain size $d$ are added. In anelasticity, frequency $f$ is added as a parameter. The four models included here take in the steady state viscosity and unrelaxed moduli in different ways. The two PsP models incorporate only $G$, while the two Xfit models incorporate $G$ and $\eta$. Furthermore, not shown, the two PsP models incorporate melt effects described by Eqn. 4 onto the pseudoperiod scaling. We incorporate the same function into the steady state viscosity in the Xfit_Maxwell method, but the Xfit_premelt method has its own method for calculating premelting effects that preclude the use of Eqn. 4.

section, we introduce a Bayesian Inference framework used to constrain $\phi, d$ and $T$ in three locales of the western U.S. The framework, however, requires mapping variations in state variables to $V_{s}$ and $Q$. While one could introduce a statistical sampling method to calculate $V_{s}$ and $Q$ over the parameter space of interest, it is sufficient for the present problem to pre-calculate a large multidimensional lookup table (LUT) as a function of $\phi, d, T, P$ and frequency $f$. The LUT can then be quickly sampled by the Bayesian Inference where needed (see following section). Furthermore, the LUT is calculated for the four anelastic methods, allowing us to compare inferred $\phi, d$ and $T$ not only between location but also between methods.

Towards that end, we used the VBRc to vary $\phi, d$ and $T$ between possible mantle values: $\phi \in(0,0.05), d \in(0.0001,0.03) \mathrm{m}, T \in(1100,1800){ }^{\circ} \mathrm{C}$ for all anelastic methods. Given the above discussion on melt-fraction, we use the poro-elastic method in all cases and include the small melt correction in the pseudoperiod and xfit Maxwell scaling methods. In Figure 7, we plot 2D slices of $Q$ and $V_{s}$ through a subset of the LUT, averaged over frequency for a single anleastic method for the Andrade pseudoperiod method. The 1D trends described above are similarly visible in the 2D slices: decreasing $V_{s}$ and $Q$ as $\phi$ increases, $d$ decreases and $T$ increases. But the 2D maps make it clear that the magnitude of the dependence changes depending on thermodynamic state; e.g., at $T=1300^{\circ} \mathrm{C}$ (top left panel), the melt fraction dependence of $V_{s}$ is stronger at smaller grain size than at larger grain size. 


\section{Application to Earth: the VBRc in a joint Bayesian frame- work}

In this section, we introduce using the VBRc within a joint Bayesian framework to place bounds on the range of state variables $\phi, T$ and $d$ likely to explain observed $V_{s}$ and $Q$. We begin with a general overview of joint Bayesian inferences in the context of the VBRc and then use the framework to compare predicted $\phi, T$ and $d$ from four representative locations of the western US.

\subsection{Joint Bayesian inference of state variables from $V_{s}\left(S_{i}\right)$ and $Q\left(S_{i}\right)$}

There are a number approaches we could use to search for the best fitting combination of state variables, $S_{i}$, to explain a measured quantity (e.g., $V_{s}(S)$ and $Q(S)$ ). One simple approach is a grid-search minimization, in which we calculate the misfit between the predicted quantities stored in the LUT described in Section 4.4 and an observed quantity in order to identify the $S_{i}$ out of all states, $S$, that minimizes the misfit. Given a multivariate input of state variables $S$, any prediction $m(S)_{\text {pred }}$ can be tested against a measurement $m_{o b s}$ with associated uncertainty $\sigma_{o b s}$ by calculating the chi-squared misfit (Eq. 30):

$$
\chi^{2}=\frac{\left(m_{o b s}-m(S)_{p r e d}\right)^{2}}{\sigma_{o b s}^{2}} .
$$

When $\chi^{2} \approx 1$, the model prediction describes the observation well.

While the grid-search minimization may be straightforward, the myriad sources of uncertainty may lead to overconfidence in results. Sources of uncertainties include both observational uncertaines in derived measurements $\left(V_{s}, Q\right)$ arising from processing the original waveform data and the uncertainty from the extrapolation of largely empirical models to fit laboratory data to mantle conditions. The Bayesian inference approach is used here instead, to provide a framework for a better sense of how well we can actually constrain state variables in the mantle from the very indirect probing by seismic waves.

Bayes' Theorem states that the posterior probability of any given state variable $s$, given a probability of the measurement $m$ is proportional to the likelihood of those $m$ values given $s$ and the prior probability of that $s$ in the first place (e.g., Bishop, 2006) :

$$
p(s \mid m)=\frac{p(m \mid s) p(s)}{p(m)} .
$$

The various probabilities have the following standard names and conceptual meanings:

- $p(s \mid m)$ : the "posterior probability", representing how well constrained the state variables of interest $s$ are given the measurements $m$. 
- $p(m \mid s)$ : the "likelihood", representing how likely the measurements are given the state variables.

- $p(s)$ : the "prior models", the probability of sampling each set of state variables.

- $p(m)$ : the "measurement probability", the probability of observing the measurement itself. In practice, $p(m)$ is not known a priori but given that it is a normalizing constant, $p(m)$ can be neglected to calculate relative probabilities, or $p(m)$ can be calculated by summing the final relative probabilities.

The likelihood, $p(m \mid s)$, comes from the $\chi^{2}$-misfit, which we use to construct a Gaussian likelihood matrix:

$$
p(m \mid s)=\frac{1}{\sqrt{2 \pi \sigma_{o b s}^{2}}} \exp \left(\frac{\chi^{2}}{2}\right)
$$

which is calculated pointwise over $S$, the multidimensional LUT of all combinations of $S$ values described in Section 4.4. Note that the combination of Eqs 30 and 32 gives the equation for a normal distribution, with the expected value equal to $m(S)_{\text {pred }}$ and the variance equal to $\sigma_{o b s}^{2}$.

The prior probability, $p(s)$, comes from our a priori knowledge of the state variables. In the present study, we start with a uniform distribution across the $\phi, T$ and $d$ parameter sweep. However, given other constraints, e.g. geothermobarometry melt- and/or xenoliths, it is possible to put in a more tightly constrained prior, with its own uncertainty, as applied below to the grain size.

\subsubsection{Uncertainty}

The benefit of Bayesian analysis is the ability to track uncertainty. However, seismic models do not include typically report measurement error. At present, we assume a minimum uncertainty of $\pm 0.05 \mathrm{~km} / \mathrm{s}$ for $V_{s}$ measurements and \pm 10 for $Q$ measurements. We also calculate an empirical standard deviation across all model points in the lateral and vertical ranges given as inputs; if this empirical uncertainty is larger, it is used for the Bayesian analysis instead. Note that we are not considering the uncertainty from experimental extrapolations, which are very hard to estimate, as they come from uncertainty in measurements as well as in fits, and then amplified over extrapolations in lengthscale (grain size) over 2-3 orders of magnitude as well as frequency.

The discussion above applies to fitting a single observation, but having both $V_{s}$ and $Q$ measurements at our disposal will influence the probable ranges of state variables $S$, where $S$ represents parameters $\phi, T$ and $d$. In this case, the initial Bayesian statement is written

$$
p\left(S \mid V_{s}, Q\right)=\frac{p\left(V_{s}, Q \mid S\right) p(S)}{p\left(V_{s}, Q\right)} .
$$

The form of the joint probability $p\left(V_{s}, Q \mid S\right)$ depends on the co- or independence of the probabilities in question. While Section 2 demonstrates the clear physics relating $V s$ and $Q$ via the state 
variables, we treat the separate observations of $V_{s}$ and $Q$ as conditionally independent in which case the total probability $p\left(V_{s}, Q \mid S\right)$ is the product of the probabilities of the separate measurements:

$$
p\left(V_{s}, Q \mid S\right)=p\left(V_{s} \mid S\right) p(Q \mid S) .
$$

Given the clear physics relating $V_{s}$ and $Q$, the assumption of conditional independence may at first seem questionable, but conditional independence in a statistical sense relates to the uncertainties in measured values $V_{s}$ and $Q$; i.e., the observation of $Q$ does not influence the uncertainty in $V_{s}$ (e.g., Dawid, 1979). Given conditional independence, the Bayesian statement of the posterior probability becomes

$$
p\left(S \mid V_{s}, Q\right)=\frac{p\left(V_{s} \mid S\right) p\left(Q \mid V_{s}\right) p(S)}{p_{o}},
$$

where we have written the $p\left(V_{s}, Q\right)$ as $p_{o}$ to emphasize its role as a normalization constant.

\subsubsection{Prior distributions of melt, temperature and (sub)grain size}

The prior models in the Bayesian approach represent pre-existing knowledge, constraints or hypotheses of the state variables. In this initial study, we treat our state variables as independent, in which case the joint prior model $p(S)$ is given as the product of the marginal probabilities (the probability of each state variable, $p(T), p(\phi), p(d)$ ). In the case of $T$ and $\phi$, we assume simple uniform $p(T)$ and $p(\phi)$ within reasonable ranges for the depth ranges chosen in our sample sights: $T \in(1100,1800)^{\circ} \mathrm{C}$ and $\phi \in(0,0.05)$. For $p(d)$ we experiment with several cases as follows.

As discussed in Section 1.2.1, there are fundamental questions in the rock mechanics community on the scaling of the HTB, in particular on the appropriate length scale for the microstructure associated with transient diffusion creep, be it the grain size or the subgrain size. To address this in a simple way across all anelastic models, we vary the Bayesian prior constraint on the grain size to test these hypotheses. For a mean stress of $\sigma_{m}=0.5 \mathrm{MPa}$, a likely level for the convecting upper mantle (and the value used in calculations here), we can estimate the mean grain size, $d_{g}$, and subgrain size, $d_{s g}$, from empirical piezometers, as $d_{g}=10$ and $\mathrm{mm} d_{s g}=1 \mathrm{~mm}$, from the Toriumi (1979) and Hirth and Kohlstedt (2015) piezometers, respectively. To test a first order dependence on grain or subgrain size, we consider the $d$ in all of the anelastic methods to be a general lengthscale and conduct three separate Bayesian experiments using different prior model probabilities for grain or subgrain size covering the range of lengthscales. In the first, we apply a uniform $p(d)$ for grain or subgrain sizes from $0.1 \mathrm{~mm}$ to $30 \mathrm{~mm}$. In the second and third case, we model $p(d)$ as a log-normal distribution with median values of $1 \mathrm{~mm}$ and $10 \mathrm{~mm}$ and standard deviation in log-space of \pm 0.25 . Thus we can see the first order effects of a subgrain or grain control by comparing the Bayesian results at these different lengthscales. 


\subsection{Application to North American upper mantle.}

In general, the seismic structure of the North American shallow upper mantle correlates with tectonic provinces, a correlation that has been observed for some time on different spatial scales. On the broadest scale, the classic study by Grand and Helmberger (1984), which remains a standard (Simmons et al., 2010), compared average velocity profiles from the "stable" North American (SNA) to those from regions within "tectonic North America" (TNA) and found that SNA exhibits a high velocity mantle lid to $200 \mathrm{~km}$ depth overlying a moderate low velocity zone until about $400 \mathrm{~km}$ depth. In contrast, TNA exhibits a strong low velocity zone from $80 \mathrm{~km}$ to about $300 \mathrm{~km}$ depth, after which velocities begin to approach those of SNA.

On a more local scale, low shear wave speeds (e.g. Rau and Forsyth, 2011) and high $V_{p} / V_{s}$ ratios (e.g., Schmandt and Humphreys, 2010) often correlate with surface volcanism, suggesting partial melting in the mantle. In contrast, the relatively amagmatic central $\mathrm{CP}$ and Wyoming Province (Tian et al., 2011; Schmandt and Humphreys, 2010; Levander et al., 2011; West et al., 2004; Lin and Ritzwoller, 2011; Xue and Allen, 2010; Wagner et al., 2010; Sigoch, 2011) are characterized by relatively high seismic velocities consistent with a dry, melt-free thermal and chemical lithosphere (e.g. Smith, 2000; Lee et al., 2001; Roy et al., 2009), but still lower than the old, undisturbed lithosphere underlying the Great Plains. Other recent studies using the USArray (TA) show similar broad features with much more detail (e.g. Yuan et al., 2011, 2014; Porter et al., 2016; Pollitz and Mooney, 2016; Calò et al., 2016).

The origin of the lateral heterogeneity on both continent and local scales is tied closely to the Cenozoic evolution of tectonics that caused extensive volcanism and lithosphere deformation throughout the tectonically active Western US. While a range of scenarios can explain the tectonic history, the end result is that western North America seems to be now riding over hotter mantle that was beneath the Pacific (Humphreys et al., 2003; Moucha et al., 2008, 2009; Liu and Gurnis, 2010), resulting in abundant regional Cenozoic magmatism, including the voluminous middleTertiary ignimbrite flare-up (Humphreys et al., 2003; Roy et al., 2009) and encroachment of magmatism on the interior of the Colorado Plateau (CP) over the past 40 Myrs (e.g. Wenrich et al., 1995; Roy et al., 2009).

\subsubsection{Measurements and Site Selection}

The present study is concerned with how the anelastic scalings may influence inferred state variables rather than comparison of seismic models, of which several detailed studies exist (e.g., Cammarano and Guerri, 2017), or geodynamic interpretations. As such, we restrict our measurements to a single velocity model and a single $Q$ model. For the velocity model, we use the 3D joint receiver function and surface wave model of Shen and Ritzwoller (2016). For the Q model, we use the global Q model of surface wave attenuation from Dalton et al. (2008).

We select three representative locations, shown in Fig. 8. The three sites are chosen along a 
single path with points chosen to reflect an expected decrease in $T$ and $\phi$ : points in the Basin and Range (BR), the Colorado Plateau (CP), and the cratonic interior east of the Rio Grande (ER). Figure 8 shows $V_{s}$ at $125 \mathrm{~km}$, cross sections of $V_{s}$ and $Q$ from the Basin and Range through the cratonic interior with the lithosphere-asthenosphere boundary and mid-lithospheric discontinuity as identified by receiver functions noted by black and white dots, respectively (Hopper and Fischer, 2018).

We calculate single measured values of $Q$ and $V_{s}$ for each site by first producing a 1D profile by averaging within a half degree at each depth resulting in the $1 \mathrm{D}$ profiles shown in figure 8 . For each site, we then select a depth range (dashed boxes in figure 9), chosen at asthenospheric depths containing the mimum in $V_{s}$ at each site. The measured values with uncertainties calculated as described in the previous section are: $V_{s}=4.14 \pm 0.053 \mathrm{~km} / \mathrm{s}$ and $Q=80 \pm 10$ for Yellowstone, $V_{s}=4.12 \pm 0.05 \mathrm{~km} / \mathrm{s}$ and $Q=54 \pm 10$ for the Basin and Range, $V_{s}=4.45 \pm 0.053 \mathrm{~km} / \mathrm{s}$ and $Q=62 \pm 10$ for the Colorado Plateau, and $V_{s}=4.61 \pm 0.053 \mathrm{~km} / \mathrm{s}$ and $Q=86 \pm 10$ for the cratonic interior.

\subsubsection{Bayesian Inference: Results}

In this section, we describe the results of the Bayesian inference. We first show the resulting probability distributions for the Basin and Range using the Andrade pseudoperiod scaling and uniform grain size prior model described in section 5.1.2, comparing separate $p\left(\phi, T, d \mid V_{s}\right)$ and $p(Q \mid \phi, T, d)$ inferences with a joint $p(\phi, T, d \mid V s, Q)$ inference. We then show the joint inference results of the log-normal grain size distributions and finally we show a comparison of $p(\phi, T \mid V s, Q)$ for all methods and all sites.

Given that the resulting probability distributions are in 3D, we present multiple marginal views of each distribution. 2D maps of the probability distributions are the probability distribution summed over the third variable that is not plotted. In Figs. 9 and 10, we also plot the marginal probability of the third variable as a line plot directly beneath each $2 \mathrm{D}$ map. Taking the first column and row of figure 10 as an example, the $2 \mathrm{D}$ plot is of $p\left(\phi, d \mid V_{s}\right)=\sum_{i} p\left(\phi, d, T_{i} \mid V_{s}\right)$ and the $1 \mathrm{D}$ plot is $p\left(T \mid V_{s}\right)=\sum_{i j} p\left(\phi_{i}, d_{j}, T \mid V_{s}\right)$. While the $2 \mathrm{D}$ plots use different colorscales to highlight features, all distributions sum to 1 as they are normalized distributions.

In order to highlight the usefulness of the joint inference, we plot result of two separate inferences on $V_{s}$ and $Q$ separately and the full joint inference, $p\left(\phi, T, d \mid V_{s}, Q\right)$, for the BR using the Andrade pseudoperiod scaling and uniform prior model on grain size in Fig. 9. In the single measurement inferences, there are generally broad distributions or bands spanning sample space, resulting in uncertain bounds of $\phi, d$ and $T$. But because the distritubtions of the separate $V_{s}$ and $Q$ measurements exhibit different trends, the joint $V_{s}, Q$ distribution ends up better constrained. Because the grain (or subgrain) dependence in $V_{s}$ and $Q$ vary, the joint distribution ends up with more narrowly confined trade off in $\phi-d$ and $T-d$ space than either measurement alone would 
provide. The joint distribution exhibits a slight preference for larger grain size visible in both the $\phi-d$ and $T-d$ distributions and the 1D marginal grain size distribution. Additionally, the most certain observation is the need for some melt given the minimal probabilities at $\phi<0.02$, visible most prominently in the $T-\phi$ and 1D marginal $\phi$ distributions.

In figure 10 we again show the joint results for the BR and Andrade pseudoperiod scaling but now for the two log-normal prior models for grain saize described in section 5.1.2. In the top and bottom rows we show the $1 \mathrm{~cm}$ and $1 \mathrm{~mm}$ median results, respectively. Because of the prior constraint on grain size, the distributions are much more tightly constrained than uniform distribution case. The likely grain sizes primarily reflect the imposed prior models, but we can clearly observe differences in $T-\phi$ space required to satisfy the observations at the different grain size distributions. The larger $1 \mathrm{~cm}$ case, more consistent with a grain size control on anelasticity, requires $T$ of around $1400^{\circ} \mathrm{C}$ and $\phi$ around 0.04 . The smaller $1 \mathrm{~mm}$ case, more consistent with a subgrain control on anelasticity, requires a much lower temperature of around $1250^{\circ} \mathrm{C}$ and interestingly still requires a similar amount of melt. The $\phi$ distribtuion in the $1 \mathrm{~mm}$ case is narrower and center at a slightly higher value than in the $1 \mathrm{~cm}$ case.

In order to compare anelastic methods, location and the influence of the grain size prior, we take the marginal melt-temperature distributions $p(\phi, T)$ and extract contours of the probability density function. In Fig. 11, we plot the 70, 80, 90 and 95\% intervals (indicated by decreasing line thickness) for the BR (orange), CP (light green) and ER (dark green) for the $1 \mathrm{~cm}$ (solid) and $1 \mathrm{~mm}$ (dashed) grain size prior models, with a panel for each anelastic method.

Comparing the method panels of Fig. 11 for the $1 \mathrm{~cm}$ case (solid curves), the different anelastic methods yield remarkably consistent distributions of $T$ and $\phi$ between sites with the exception of the Maxwell normalization. The andrade_psp, eburgers_psp and xfit_premelt methods all show the Basin and Range distribution (orange) centered at $\phi$ between 0.03-0.04 and $T$ from $1400-1500^{\circ} \mathrm{C}$, the Colorado Plateau distribution (light green) centered at $\phi \approx 0.005-0.01$ and $T \approx 1500^{\circ} \mathrm{C}$, and the cratonic interior (dark green) centered at $\phi=0$ with $T \approx 1400^{\circ} \mathrm{C}$. The Maxwell scaling produces much wider distributions, though the general relative position of the distribution centers are in the same order as the other anelastic methods. For the smaller $1 \mathrm{~mm}$ prior constraint, Fig. 11 exhibits lower $T$ ranges and larger differences between anelastic methods. The distributions for the andrade_psp and eburgers_psp methods shift lower in $T$ by about $100^{\circ} \mathrm{C}$ while both xfit methods shift more drastically. Interestingly the shift is mostly along the $T$ axis and the $\phi$ ranges are relatively unchanged.

Finally, we calculate the ensemble probability distributions across models for each site, given by the weighted sum of the distributions. For simplicity, we assume equal weighting of models (e.g. Watterson, 2008) though a more complete treatment could calculate weights based on uncertainty contained within each model (e.g. Min et al., 2009). In Fig. 12 we show two cases: in the left and right columns, we show ensemble distributions for the $1 \mathrm{~cm}$ and $1 \mathrm{~mm}$ (sub)grain size prior model cases, respectively. While the distributions of the ensemble plots are broader and 
more complex than those of the individual methods, the general trend of increasing melt fraction from BR to CP to ER is still discernible. In the case of the $1 \mathrm{~mm}$ ensemble, differences in the xfit and pseudoperiod methods at the lower grain/subgrain scale result in lobed distributions for $\mathrm{CP}$ and ER at high and low temperatures. As we discuss below, these ensemble plots give us a sense for how confident we can be in our inferred $T$ and $\phi$ ranges if we are agnostic towards anelastic method.

\subsection{Discussion}

Our primary aim in this paper is demonstrate the methods and uncertainties, but not to push far into the interpretation in a geodynamic context. However, the results of the Bayesian inference do have some intriguing aspects both for geodynamics and for the rock physics questions, even in light of the relatively large uncertainties in the Bayesian distributions.

Turning first to geodynamic implications, one of the interesting results of the Bayesian inference is the lack of a clear positive trend in temperature and melt fraction moving from ER to $\mathrm{CP}$ to $\mathrm{BR}$ as one might initially expect for these regions. Considering the ensemble plots of Fig. 12, which capture uncertainty in anelastic method when we do not impose a preferred method, the clearest signal is an increase in likely melt fraction from ER to CP to BR. When looking at individual methods (Fig. 11), the xfit_mxw method exhibits a positive trend in $T-\phi$, with the remaining methods exhibiting lower to no positive trends. The lack of a clear $T-\phi$ trend is perhaps at first surprising, but less so when we relax our expectation that equilibrium petrologic relationships between temperature and melt fraction apply to melt migration in the asthenosphere where the physical melt fraction (porosity) may not match the thermodynamic melt fraction (degree of melting).

While temperature controls whether or not there can be melt, the actual melt fraction measured by seismic waves is controlled by the rate of melt production and the physics of melt migration. Melt production in the asthenosphere generally occurs by adiabatic decompression melting and so the melting rate is ultimately modulated by the asthenosphere upwelling rate in addition to bulk composition, volatile content and temperature (e.g., Phipps Morgan, 2001; Hewitt, 2010). Additionally, a number of coupled processes related to melt migration including chemical (e.g., Daines and Kohlstedt, 1994; Aharonov et al., 1995; Pec et al., 2020) and mechanical (e.g., Stevenson, 1989; Holtzman et al., 2003) instabilities, permeability barriers (e.g., Sparks and Parmentier, 1991; Havlin et al., 2013) and pressure gradients arising from solid deformation (e.g., Spiegelman and McKenzie, 1987; Roy et al., 2016) will act to redistribute melt. So ultimately the melt distribution sampled by seismic waves will arise from the complex interplay of all these processes. But if we take our ensemble results at face value, one simple interpretation is that the asthenosphere temperature at all these locations is similar and the melt production decreases from $\mathrm{BR}$ to $\mathrm{CP}$ to $\mathrm{ER}$, perhaps reflecting a transition from larger scale convective motion in the BR to small scale 
convection beneath the $\mathrm{CP}$ to effectively no asthenosphere upwelling beneath the ER. Currently in the BR, active extension likely controls upwelling in the asthenosphere while upwelling beneath the CP is likely dominated by small-scale convective processes (e.g., Ballmer et al., 2015; Roy et al., 2016) and delamination (e.g., Levander et al., 2011), which would result in lower upwelling rate and thus melt production rate beneath the $\mathrm{CP}$ even at the same asthenosphere temperature. Thus, the lack of a positive $T-\phi$ trend may simply imply that the asthenosphere is at a similar temperature across the western U.S. and $\phi$ is controlled more by the production and migration of melt in different convective regimes.

The results of the Bayesian inference also lends some insight into some open questions in the rock physics community, in particularly on the question of grain or subgrain control on anelasticity. When using a uniform prior distribution on grain or subgrain size, the joint probability distributions are quite broad, showing a weak preference for grain or subgain sizes greater than 1 mm (left columns of Fig. 9), with slightly narrower melt fraction-temperature tradeoffs. When a strong prior on grain size is imposed, the range of probable temperature and melt fraction values narrows substantially, as shown in Fig. 10 for the Andrade_PsP but the different sensitivities of each anelastic method to grain size leads to larger differences at the smaller grain size. This is visible in Fig. 11, in which the Xfit_premelt and eBurgers_PsP models give very similar results at $d=1 \mathrm{~cm}$, but differ by large margins at $d=1 \mathrm{~mm}$. In the case of the Xfit premelt method, the temperature ranges at the $d=1 \mathrm{~mm}$ case are likely too low in order to balance the strength of the pre-melting effect and maintain the observed $Q$ and $V_{s}$. A smaller grain size dependence (with a grain size exponent closer to that in the eBurgers_PsP scaling) would minimize this difference.

In terms of absolute temperatures, the prior model for a $1 \mathrm{~mm}$ subgrain control does yield temperatures that seem more reasonable than the $1 \mathrm{~cm}$ case, suggesting a general preference for subgrain control: at $1 \mathrm{~mm}$, the eBurgers_PsP and and xfit_mxw methods yield temperature ranges spanning those inferred by joint seismic and petrologic inversions in the Basin and Range (Plank and Forsyth, 2016) who found likely potential temperatures from 1280 to $1525^{\circ} \mathrm{C}$ (or about 1330 to $1472^{\circ} \mathrm{C}$ in absolute temperatures at the depth ranges considered here). Furthermore, these findings that a $1 \mathrm{~cm}$ grain size predicts a $Q$ that is too high, requiring higher temperatures to match observed values of $Q$ and $V_{s}$, are consistent with the extrapolation of Gribb and Cooper (1998) and inference of Abers et al. (2014) that required significant weakening above the HTB to explain measurements in subduction wedges.

Finally, the Bayesian analysis here could be improved in a number of ways. Including additional prior information derived from other sources such as magnetotelluric inversions, xenolith studies or petrology would also narrow the distributions. And of course, compositional variation and their effects on elastic, viscous and anelastic properties may also play a significant role. Volatiles are a particularly challenging but critical piece to address. In addition to possible direct effects of water on the high temperature background (see Sec. 1.2.4), the pre-melting scaling implies an interesting coupling with melt generation and transport. If $\mathrm{Q}$ varies with the solidus, then 
Q should vary systematically with volatile variations both between regions with varying volatile content and within the melting column in a single region. The fact that many of the variables controlling melt production and migration overlap with those that control anelasticity suggests that a more complete forward modeling approach that includes melt generation and migration with volatile transport is needed in order to more self-consistently infer the thermodynamic state. Ultimately, the inference is only as good as the seismic measurements, and also the physics contained in the constitutive models and how closely they represent and capture the processes occurring in the Earth. But the Bayesian inference framework provides an enormously valuable tool for systematically tracking how well we can actually know the thermodynamic state given the knowledge at hand.

\section{Conclusions}

The Very Broadband Rheology Calculator provides a useful way of building a statistical framework to quantify uncertainty in seismic properties arising from different anelastic methods, in a forward sense. In the context of a Bayesian inference of thermodynamic state, the VBRc tells us how well we can constrain any set of state state variables given uncertainty in the seismic measurements. Along with many rapid advancements, many open problems in the rock physics understanding exist and can be integrated into constitutive models implemented in the VBRc as a community tool.

\section{Acknowledgements}

This work has been supported by a series of NSF grants to B. Holtzman, in particular, NSF Geophysics (EAR 1056332): CAREER: Very Broadband Rheology and the Internal Dynamics of Plate Boundaries, and NSF Earthscope (EAR 1736165, co-I Havlin): Mapping variability in the thermo-mechanical structure of the North American Plate and upper mantle, as well as NSF Geophysics (EAR 13-15254, PI-J. Davis). We thank R. Cooper and U. Faul for very constructive reviews. The Very Broadband Rheology Calculator is available for download and use at https://vbr-calc.github.io/vbr/ .

\section{References}

Abers, G.A., Fischer, K., Hirth, G., Wiens, D., Plank, T., Holtzman, B.K., McCarthy, C., Gazel, E., 2014. Reconciling mantle attenuation-temperature relationships from seismology, petrology, and laboratory measurements. Geochemistry, Geophysics, Geosystems 15, 3521-3542.

Accardo, N., Gaherty, J., Shillington, D., Hopper, E., Nyblade, A., Ebinger, C., Scholz, C., Chindandali, P., Wambura-Ferdinand, R., Mbogoni, G., et al., 2020. Thermochemical modification 
of the upper mantle beneath the northern malawi rift constrained from shear velocity imaging. Geochemistry, Geophysics, Geosystems 21, e2019GC008843.

Afonso, J.C., Fernandez, M., Ranalli, G., Griffin, W., Connolly, J., 2008. Integrated geophysicalpetrological modeling of the lithosphere and sublithospheric upper mantle: Methodology and applications. Geochemistry, Geophysics, Geosystems 9.

Aharonov, E., Whitehead, J.A., Kelemen, P.B., Spiegelman, M., 1995. Channeling instability of upwelling melt in the mantle. J. Geophys. Res. 100, 20433-20450. URL: http: / dx . doi . org/10.1029/95JB01307.

Aizawa, Y., Barnhoorn, A., Faul, U.H., Gerald, J.D.F., Jackson, I., Kovács, I., 2008. Seismic properties of anita bay dunite: an exploratory study of the influence of water. Journal of Petrology 49, 841-855.

Andrade, E.N.d., 1962. On the validity of $t^{1 / 3}$ Law of Flow of Metals. Philosophical Magazine 7, 2003-\&.

Andrade, E.N.d.C., 1910. On the Viscous Flow in Metals, and Allied Phenomena. Proceedings of the Royal Society A: Mathematical, Physical and Engineering Sciences 84, 1-12.

Ballmer, M.D., Conrad, C.P., Smith, E.I., Johnsen, R., 2015. Intraplate volcanism at the edges of the colorado plateau sustained by a combination of triggered edge-driven convection and shear-driven upwelling. Geochemistry, Geophysics, Geosystems 16, 366-379.

Behn, M.D., Hirth, G., Ii, J.R.E., 2009. Implications of grain size evolution on the seismic structure of the oceanic upper mantle. Earth and Planetary Science Letters 282, 178-189. doi:10.1016/j.epsl.2009.03.014.

Bellis, C., Holtzman, B., 2014. Sensitivity of seismic measurements to frequency-dependent attenuation and upper mantle structure: an initial approach. Journal of Geophysical Research doi:10.1002/2013JB010831.

Bishop, C.M., 2006. Pattern recognition and machine learning. springer.

Budiansky, B., Sumner, E.E., O'Connell, R.J., 1983. Bulk thermoelastic attenuation of composite materials. J. Geophys. Res. 88, 10-343-10-348.

Byrnes, J.S., Bezada, M., Long, M.D., Benoit, M.H., 2019. Thin lithosphere beneath the central appalachian mountains: constraints from seismic attenuation beneath the magic array. Earth and Planetary Science Letters 519, 297-307. 
Calò, M., Bodin, T., Romanowicz, B., 2016. Layered structure in the upper mantle across north america from joint inversion of long and short period seismic data. Earth and Planetary Science Letters 449, 164-175.

Cammarano, F., Goes, S., Vacher, P., Giardini, D., 2003. Inferring upper-mantle temperatures from seismic velocities. Physics of The Earth and Planetary Interiors 138, 197-222. doi: 10 . $1016 / \mathrm{s} 0031-9201(03) 00156-0$.

Cammarano, F., Guerri, M., 2017. Global thermal models of the lithosphere. Geophysical Journal International 210, 56-72.

Cammarano, F., Romanowicz, B., Stixrude, L., Lithgow-Bertelloni, C., Xu, W., 2009. Inferring the thermochemical structure of the upper mantle from seismic data. Geophysical Journal International 179, 1169-1185.

Chrysochoos, A., 2012. Thermomechanical analysis of the cyclic behavior of materials. Procedia Iutam 4, 15-26.

Cline II, C., Faul, U., David, E., Berry, A., Jackson, I., 2018. Redox-influenced seismic properties of upper-mantle olivine. Nature 555, 355-358.

Cobden, L., Goes, S., Cammarano, F., Connolly, J.A., 2008. Thermochemical interpretation of one-dimensional seismic reference models for the upper mantle: evidence for bias due to heterogeneity. Geophysical Journal International 175, 627-648.

Connolly, J., 2005. Computation of phase equilibria by linear programming: A tool for geodynamic modeling and its application to subduction zone decarbonation. Earth and Planetary Science Letters 236, 524-541. doi:10.1016/j.eps 1.2005.04.033.

Cooper, R.F., 2002. Seismic Wave Attenuation: Energy Dissipation in Viscoelastic Crystalline Solids . Reviews in Mineralogy and Geochemistry 51, 253-290.

Cukjati, J.T., Cooper, R.F., Parman, S.W., Zhao, N., Akey, A.J., Laiginhas, F.A., 2019. Differences in chemical thickness of grain and phase boundaries: an atom probe tomography study of experimentally deformed wehrlite. Physics and Chemistry of Minerals 46, 845-859.

Daines, M.J., Kohlstedt, D.L., 1994. The transition from porous to channelized flow due to melt/rock reaction during melt migration. Geophys. Res. Lett. 21, 145-148. URL: http: //dx.doi.org/10.1029/93GL03052.

Dalton, C.A., Ekström, G., Dziewoński, A.M., 2008. The global attenuation structure of the upper mantle. Journal of Geophysical Research: Solid Earth 113. 
Dalton, C.A., Faul, U.H., 2010. The oceanic and cratonic upper mantle: Clues from joint interpretation of global velocity and attenuation models. Lithos 120, 160-172.

Dawid, A.P., 1979. Conditional independence in statistical theory. Journal of the Royal Statistical Society: Series B (Methodological) 41, 1-15.

Duffy, T.S., Anderson, D.L., 1989. Seismic velocities in mantle minerals and the mineralogy of the upper mantle. Journal of Geophysical Research: Solid Earth 94, 1895-1912.

Eaton, J.W., Bateman, D., Hauberg, S., Wehbring, R., 2015. GNU Octave version 4.0.0 manual: a high-level interactive language for numerical computations. URL: http: //www. gnu . org/software/octave/doc/interpreter.

Farla, R.J., Jackson, I., Gerald, J.D.F., Faul, U.H., Zimmerman, M.E., 2012. Dislocation damping and anisotropic seismic wave attenuation in earth's upper mantle. Science 336, 332-335.

Faul, U., Fitzgerald, J., Jackson, I., 2004. Shear wave attenuation and dispersion in melt-bearing olivine polycrystals: 2 . Microstructural interpretation and seismological implications. J Geophys Res-Sol Ea 109, B06202.

Faul, U., Jackson, I., 2007. Diffusion creep of dry, melt-free olivine. Journal of Geophysical Research 112, 2341-2344. doi:10.1029/2006 JB004586.

Faul, U., Jackson, I., 2015. Transient creep and strain energy dissipation: An experimental perspective. Annual Review of Earth and Planetary Sciences 43, 541-569.

Goes, S., Govers, R., Vacher, P., 2000. Shallow mantle temperatures under europe from p and s wave tomography. Journal of Geophysical Research: Solid Earth 105, 11153-11169.

Goes, S., van der Lee, S., 2002. Thermal structure of the north american uppermost mantle inferred from seismic tomography. Journal of Geophysical Research 107.

Grand, S.P., Helmberger, D.V., 1984. Upper mantle shear structure of north america. Geophysical Journal International 76, 399-438.

Gribb, T., Cooper, R., 1998. Low-frequency shear attenuation in polycrystalline olivine: Grain boundary diffusion and the physical significance of the Andrade model for viscoelastic rheology. Journal Of Geophysical Research-Solid Earth 103, 27,267-27,279.

Gribb, T., Cooper, R., 2000. The effect of an equilibrated melt phase on the shear creep and attenuation behavior of polycrystalline olivine. Geophys. Res. Lett. 27, 2341-2344.

Gueguen, Y., Darot, M., Mazot, P., Woirgard, J., 1989. Q- 1 of forsterite single crystals. Physics of the earth and planetary interiors 55, 254-258. 
Hacker, B., Abers, G., Peacock, S., 2003. Subduction factory 1. theoretical mineralogy, densities, seismic wave speeds, and $\mathrm{H}_{2} \mathrm{O}$ contents. J. Geophys. Res 108, 2029.

Hammond, W., Humphreys, E., 2000. Upper mantle seismic wave attenuation: Effects of realistic partial melt distribution. J Geophys Res-Sol Ea 105, 10987-10999.

Hansen, L.N., Zimmerman, M.E., Kohlstedt, D.L., 2011. Grain boundary sliding in san carlos olivine: Flow law parameters and crystallographic-preferred orientation. J Geophys Res-Sol Ea 116, B08201. doi:10.1029/2011JB008220.

Havlin, C., Holtzman, B., Hopper, E., 2020. Very broadband rheology calculator URL: https : //doi.org/10.5281/zenodo.4317821, doi:10.5281/zenodo.4317821.

Havlin, C., Parmentier, E., Hirth, G., 2013. Dike propagation driven by melt accumulation at the lithosphere-asthenosphere boundary. Earth and Planetary Science Letters 376, 20-28. doi:10 . $1016 / j . e p s 1.2013 .06 .010$.

Hewitt, I.J., 2010. Modelling melting rates in upwelling mantle. Earth and Planetary Science Letters 300, 264-274.

Hier-Majumder, S., 2008. Influence of contiguity on seismic velocities of partially molten aggregates. Journal of Geophysical Research 113, 14. doi:10 .1029/2008 JB005662.

Hier-Majumder, S., Drombosky, T., 2015. Development of anisotropic contiguity in deforming partially molten aggregates: 2 . implications for the lithosphere-asthenosphere boundary. Journal of Geophysical Research: Solid Earth 120, 764-777.

Hirth, G., Kohlstedt, D.L., 1995a. Experimental constraints on the dynamics of the partially molten upper mantle 2. Deformation in the dislocation creep regime. J. Geophys. Res. B8, $15,441-15,449$.

Hirth, G., Kohlstedt, D.L., 1995b. Experimental constraints on the dynamics of the partially molten upper mantle: Deformation in the diffusion creep regime. J. Geophys. Res. 100, 19812001.

Hirth, G., Kohlstedt, D.L., 2003. Rheology of the upper mantle and the mantle wedge: A view from the experimentalists. Geophysical Monograph Series 138, 83-105.

Hirth, G., Kohlstedt, D.L., 2015. The stress dependence of olivine creep rate: Implications for extrapolation of lab data and interpretation of recrystallized grain size. Earth and Planetary Science Letters 418, 20-26. 
Hoggard, M.J., Czarnota, K., Richards, F.D., Huston, D.L., Jaques, A.L., Ghelichkhan, S., 2020. Gigayear stability of cratonic edges controls global distribution of sediment-hosted metals. Nature Geoscience 13, 7.

Holtzman, B.K., 2016. Questions on the existence, persistence, and mechanical effects of a very small melt fraction in the asthenosphere. Geochemistry, Geophysics, Geosystems 17, 470-484.

Holtzman, B.K., Groebner, N., Zimmerman, M.E., Ginsberg, S.B., Kohlstedt, D.L., 2003. Stressdriven melt segregation in partially molten rocks. Geochem. Geophys. Geosyst. 4, 26. doi:10 . $1029 / 2001 \mathrm{GC} 000258$.

Hopper, E., Fischer, K.M., 2018. The changing face of the lithosphere-asthenosphere boundary: Imaging continental scale patterns in upper mantle structure across the contiguous u.s. with sp converted waves. Geochem. Geophys. Geosyst. 19, 2593-2614. URL: https : / / doi .org/ $10.1029 / 2018 \mathrm{GC} 007476$, doi:10.1029/2018GC007476.

Hopper, E., Gaherty, J.B., Shillington, D.J., Accardo, N.J., Nyblade, A.A., Holtzman, B.K., Havlin, C., Scholz, C.A., Chindandali, P.R., Ferdinand, R.W., et al., 2020. Preferential localized thinning of lithospheric mantle in the melt-poor malawi rift. Nature Geoscience 13, 584-589.

Humphreys, E.D., Hessler, E., Dueker, K., Farmer, G.L., Erslev, E., Atwater, T., 2003. How laramide-age hydration of north american lithosphere by the farallon slab controlled subsequent activity in the western united states. International Geology Review 45, 575-595.

Isaak, D.G., 1992. High-temperature elasticity of iron-bearing olivines. Journal of Geophysical Research: Solid Earth 97, 1871-1885.

Jackson, I., 2007. Properties of Rocks and Minerals - Physical Origins of Anelasticity and Attenuation in Rock, in: Schubert, G. (Ed.), Treatise on Geophysics. Elsevier, pp. 493-525.

Jackson, I., Faul, U., Gerald, J., Morris, S., 2006. Contrasting viscoelastic behavior of meltfree and melt-bearing olivine: Implications for the nature of grain-boundary sliding. Materials Science and Engineering: A 442,170-174. doi:10.1016/j.msea.2006.01.136.

Jackson, I., Faul, U.H., 2010. Grainsize-sensitive viscoelastic relaxation in olivine: Towards a robust laboratory-based model for seismological application. Physics of The Earth and Planetary Interiors 183, 151-163.

Jackson, I., Faul, U.H., Fitz Gerald, J.D., Tan, B.H., 2004. Shear wave attenuation and dispersion in melt-bearing olivine polycrystals: 1 . specimen fabrication and mechanical testing. Journal of Geophysical Research: Solid Earth 109. 
Jackson, I., Faul, U.H., Skelton, R., 2014. Elastically accommodated grain-boundary sliding: New insights from experiment and modeling. Physics of the Earth and Planetary Interiors 228, 203-210.

Karato, S.i., 2012. On the origin of the asthenosphere. Earth and Planetary Science Letters 321, 95-103.

Katz, R.F., 2003. A new parameterization of hydrous mantle melting. Geochem. Geophys. Geosyst. 4, 19. doi:10.1029/2002GC000433.

Khan, A., Boschi, L., Connolly, J.A.D., 2009. On mantle chemical and thermal heterogeneities and anisotropy as mapped by inversion of global surface wave data. J Geophys Res 114, B09305. doi:10.1029/2009JB006399.

Khan, A., Zunino, A., Deschamps, F., 2011. The thermo-chemical and physical structure beneath the north american continent from bayesian inversion of surface-wave phase velocities. Journal of Geophysical Research 116. doi:10.1029/2011JB008380.

Kohlstedt, D., Hansen, L., 2015. Properties of rocks and minerals - constitutive equations, rheological behavior, and viscosity of rocks. Treatise on Geophysics 2.18, 441-472.

Kumazawa, M., Anderson, O.L., 1969. Elastic moduli, pressure derivatives, and temperature derivatives of single-crystal olivine and single-crystal forsterite. J. Geophys. Res. 74, 5961-\&.

Lau, H.C., Holtzman, B.K., 2019. "measures of dissipation in viscoelastic media" extended: Toward continuous characterization across very broad geophysical time scales. Geophysical Research Letters 46, 9544-9553.

Lau, H.C., Holtzman, B.K., Havlin, C., 2020. Toward a self-consistent characterization of lithospheric plates using full-spectrum viscoelasticity. AGU Advances 1, e2020AV000205.

Lee, C.T., Yin, Q., Rudnick, R.L., Jacobsen, S.B., 2001. Preservation of ancient and fertile lithospheric mantle beneath the southwestern united states. Nature 411, 69-73.

Lee, C.T.A., 2003. Compositional variation of density and seismic velocities in natural peridotites at stp conditions: Implications for seismic imaging of compositional heterogeneities in the upper mantle. Journal of Geophysical Research: Solid Earth 108.

Levander, A., Schmandt, B., Miller, M.S., Liu, K., Karlstrom, K.E., Crow, R.S., Lee, C.T.A., Humphreys, E.D., 2011. Continuing colorado plateau uplift by delamination-style convective lithospheric downwelling. Nature 472, 461.

Lin, F.C., Ritzwoller, M.H., 2011. Helmholtz surface wave tomography for isotropic and azimuthally anisotropic structure. Geophysical Journal International 186, 1104-1120. 
Liu, L., Gurnis, M., 2010. Dynamic subsidence and uplift of the colorado plateau. Geology 38, 663-666.

MATLAB, 2017. version 9.2.0 (R2017a). The MathWorks Inc., Natick, Massachusetts.

Mavko, G., Nur, A., 1975. Melt squirt in the asthenosphere. Journal of Geophysical Research 80, 1444-1448.

McCarthy, C., Cooper, R.F., 2016. Tidal dissipation in creeping ice and the thermal evolution of europa. Earth and Planetary Science Letters 443, 185-194.

McCarthy, C., Takei, Y., 2011. Anelasticity and viscosity of partially molten rock analogue: Toward seismic detection of small quantities of melt. Geophysical Research Letters 38, L18306.

McCarthy, C., Takei, Y., Hiraga, T., 2011. Experimental study of attenuation and dispersion over a broad frequency range: 2 . The universal scaling of polycrystalline materials. Journal Of Geophysical Research-Solid Earth 116, B09207.

Min, Y.M., Kryjov, V.N., Park, C.K., 2009. A probabilistic multimodel ensemble approach to seasonal prediction. Weather and Forecasting 24, 812-828.

Minster, J.B., Anderson, D.L., 1980. Dislocations and nonelastic processes in the mantle. Journal of Geophysical Research: Solid Earth 85, 6347-6352.

Morris, S., Jackson, I., 2009. Diffusionally assisted grain-boundary sliding and viscoelasticity of polycrystals. Journal Of The Mechanics And Physics Of Solids 57, 744-761.

Moucha, R., Forte, A.M., Rowley, D.B., Mitrovica, J.X., Simmons, N.A., Grand, S.P., 2008. Mantle convection and the recent evolution of the colorado plateau and the rio grande rift valley. Geology 36, 439-442.

Moucha, R., Forte, A.M., Rowley, D.B., Mitrovica, J.X., Simmons, N.A., Grand, S.P., 2009. Deep mantle forces and the uplift of the colorado plateau. Geophysical Research Letters 36.

O’Connell, R., Budiansky, B., 1977. Viscoelastic properties of fluid-saturated cracked solids. J. Geophys. Res. 82, 5719-5735.

Pec, M., Holtzman, B., Zimmerman, M., Kohlstedt, D.L., 2020. Influence of lithology on reactive melt flow channelization. Geochemistry, Geophysics, Geosystems 21, e2020GC008937.

Phipps Morgan, J., 2001. Thermodynamics of pressure release melting of a veined plum pudding mantle. Geochemistry, Geophysics, Geosystems 2. 
Plank, T., Forsyth, D.W., 2016. Thermal structure and melting conditions in the mantle beneath the basin and range province from seismology and petrology. Geochemistry, Geophysics, Geosystems 17, 1312-1338.

Pollitz, F.F., Mooney, W.D., 2016. Seismic velocity structure of the crust and shallow mantle of the central and eastern united states by seismic surface wave imaging. Geophysical Research Letters 43, 118-126.

Porter, R., Liu, Y., Holt, W.E., 2016. Lithospheric records of orogeny within the continental us. Geophysical Research Letters 43, 144-153.

Priestley, K., McKenzie, D., 2013. The relationship between shear wave velocity, temperature, attenuation and viscosity in the shallow part of the mantle. Earth and Planetary Science Letters $381,78-91$.

Raj, R., 1975. Transient behavior of diffusion-induced creep and creep rupture. Metallurgical and Materials Transactions A 6A, 1499-1509.

Raj, R., Ashby, M., 1971. On Grain Boundary Sliding and Diffusional Creep. Metallurgical Transactions 2, 1113-1127.

Rau, C.J., Forsyth, D.W., 2011. Melt in the mantle beneath the amagmatic zone, southern nevada. Geology 39, 975-978.

Rempel, A., Wettlaufer, J., Worster, M., 2001. Interfacial premelting and the thermomolecular force: thermodynamic buoyancy. Physical review letters 87, 088501.

Richards, F.D., Hoggard, M.J., White, N.J., Ghelichkhan, S., 2020. Exploring the relationship between upper mantle structure and short wavelength dynamic topography using calibrated anelasticity parameterizations. in review, Journal of Geophysical Research: Solid Earth .

Roy, M., Gold, S., Johnson, A., Osuna Orozco, R., Holtzman, B.K., Gaherty, J., 2016. Macroscopic coupling of deformation and melt migration at continental interiors, with applications to the colorado plateau. Journal of Geophysical Research: Solid Earth 121, 3762-3781.

Roy, M., Jordan, T.H., Pederson, J., 2009. Colorado plateau magmatism and uplift by warming of heterogeneous lithosphere. Nature 459, 978-982. doi:10.1038/nature08052.

Rudge, J.F., 2018. The viscosities of partially molten materials undergoing diffusion creep. Journal of Geophysical Research: Solid Earth 123, 10-534.

Sasaki, Y., Takei, Y., McCarthy, C., Rudge, J.F., 2019. Experimental study of dislocation damping using a rock analogue. Journal of Geophysical Research: Solid Earth 124, 6523-6541. 
Schmandt, B., Humphreys, E., 2010. Complex subduction and small-scale convection revealed by body-wave tomography of the western united states upper mantle. Earth and Planetary Science Letters 297, 435-445. doi:10.1016/j.eps . 2010.06 .047$.

Schmeling, H., 1985. Numerical models on the influence of partial melt on elastic, anelastic and electric properties of rocks. part i: elasticity and anelasticity. Physics of the earth and planetary interiors $41,34-57$.

Schutt, D.L., Lesher, C.E., 2006. Effects of melt depletion on the density and seismic velocity of garnet and spinel lherzolite. J. Geophys. Res. 111, 24. doi:10.1029/2003 JB002950.

Shen, W., Ritzwoller, M.H., 2016. Crustal and uppermost mantle structure beneath the united states. Journal of Geophysical Research: Solid Earth 121, 4306-4342.

Sigoch, K., 2011. Mantle provinces under north america from multifrequency p wave tomography. Geochemistry, Geophysics, Geosystems 12.

Simmons, N.A., Forte, A.M., Boschi, L., Grand, S.P., 2010. Gypsum: A joint tomographic model of mantle density and seismic wave speeds. Journal of Geophysical Research: Solid Earth 115.

Smith, D., 2000. Insights into the evolution of the uppermost continental mantle from xenolith localities on and near the Colorado Plateau and regional comparisons. Journal of Geophysical Research 105, 16769-16781.

Sparks, D., Parmentier, E., 1991. Melt extraction from the mantle beneath spreading centers. Earth and Planetary Science Letters 105, 368-377.

Spiegelman, M., McKenzie, D., 1987. Simple 2-d models for melt extraction at mid-ocean ridges and island arcs. Earth and Planetary Science Letters 83, 137-152.

Stevenson, D., 1989. Spontaneous small-scale melt segregation in partial melts undergoing deformation. Geophys. Res. Lett. 16, 1067-1070.

Stixrude, L., 2007. Properties of rocks and minerals - seismic properties of rocks and minerals, and structure of the earth. Treatise on Geophysics 2.02, 1-26.

Stixrude, L., Lithgow-Bertelloni, C., 2005. Mineralogy and elasticity of the oceanic upper mantle: Origin of the low-velocity zone. J Geophys Res-Sol Ea 110, B03204. doi:10.1029/ 2004 JB002965.

Sundberg, M., Cooper, R., 2010. A composite viscoelastic model for incorporating grain boundary sliding and transient diffusion creep: Correlating creep and attenuation responses for materials with a fine grain size. Philos Mag 90, 2817-2840. 
Takei, Y., 1998. Constitutive mechanical relations of solid-liquid composites in terms of grainboundary contiguity. J. Geophys. Res. 103, 18,183-18,203.

Takei, Y., 2002. Effect of pore geometry on Vp/Vs: From equilibrium geometry to crack. J. Geophys. Res. 107, 2043. doi:10.1029/2001 JB000522.

Takei, Y., 2013. Elasticity, anelasticity, and viscosity of a partially molten rock. Physics and Chemistry of the Deep Earth (S.i. Karato, ed.) 66, 93.

Takei, Y., 2017. Effects of partial melting on seismic velocity and attenuation: A new insight from experiments. Annual Review of Earth and Planetary Sciences 45, 447-470.

Takei, Y., 2019. Phase-field modeling of grain boundary premelting. Journal of Geophysical Research: Solid Earth 124, 8057-8076.

Takei, Y., Holtzman, B.K., 2009a. Viscous constitutive relations of solid-liquid composites in terms of grain boundary contiguity: 1. Grain boundary diffusion control model. J. Geophys. Res. 114, 19. doi:10.1029/2008 JB005850.

Takei, Y., Holtzman, B.K., 2009b. Viscous constitutive relations of solid-liquid composites in terms of grain boundary contiguity: 2 . Compositional model for small melt fractions. J. Geophys. Res. 114, 18. doi:10.1029/2008 JB005851.

Takei, Y., Karasawa, F., Yamauchi, H., 2014. Temperature, grain size, and chemical controls on polycrystal anelasticity over a broad frequency range extending into the seismic range. Journal of Geophysical Research: Solid Earth 119, 5414-5443.

Tian, Y., Zhou, Y., Sigloch, K., Nolet, G., Laske, G., 2011. Structure of north american mantle constrained by simultaneous inversion of multiple-frequency sh, ss, and love waves. Journal of Geophysical Research: Solid Earth 116. URL: http://dx.doi.org/10.1029/ 2010 JB007704, doi:10.1029/2010JB007704.

Toriumi, M., 1979. Relation between dislocation density and subgrain size of naturally deformed olivine in peridotites. Contributions to mineralogy and petrology 68, 181-186.

Wagner, L., Forsyth, D.W., Fouch, M.J., James, D.E., 2010. Detailed three-dimensional shear wave velocity structure of the northwestern united states from rayleigh wave tomography. Earth and Planetary Science Letters 299.

Watterson, I.G., 2008. Calculation of probability density functions for temperature and precipitation change under global warming. Journal of Geophysical Research: Atmospheres 113. 
Wenrich, K.J., Billingsley, G.H., Blackerby, B.A., 1995. Spatial migration and compositional changes of Miocene-Quaternary magmatism in the western Grand Canyon. Journal of Geophysical Research 100, 10417-10440.

West, M., Ni, J., Baldridge, W., Wilson, D., Aster, R., Gao, W., Grand, S., 2004. Crust and upper mantle shear-wave structure of the southwest United States: Implications for rifting and support for high elevation. Journal of Geophysical Research 109.

Xu, Y., Zimmerman, M.E., Kohlstedt, D.L., 2004. Deformation behavior of partially molten mantle rocks. Rheology and deformation of the lithosphere at continental margins eds. Garry D. Karner, Brian Taylor, Neal W. Driscoll, 27.

Xue, M., Allen, R.M., 2010. Mantle structure beneath the western united states and its implications for convection processes. Journal of Geophysical Research B07303.

Yamauchi, H., Takei, Y., 2016. Polycrystal anelasticity at near-solidus temperatures. Journal of Geophysical Research: Solid Earth .

Yuan, H., French, S., Cupillard, P., Romanowicz, B., 2014. Lithospheric expression of geological units in central and eastern north america from full waveform tomography. Earth and Planetary Science Letters 402, 176-186.

Yuan, H., Romanowicz, B., Fischer, K.M., Abt, D., 2011. 3-d shear wave radially and azimuthally anisotropic velocity model of the north american upper mantle. Geophysical Journal International 184, 1237-1260.

Zener, C., 1941. Theory of the elasticity of polycrystals with viscous grain boundaries. Physical Review 60, 906.

Zhao, N., Hirth, G., Cooper, R.F., Kruckenberg, S.C., Cukjati, J., 2019. Low viscosity of mantle rocks linked to phase boundary sliding. Earth and Planetary Science Letters 517, 83-94.

Zhou, Y., 2009. Surface-wave sensitivity to 3-d anelasticity. Geophysical Journal International $178,1403-1410$. 
(a) high temperature background (HTB) attenuation
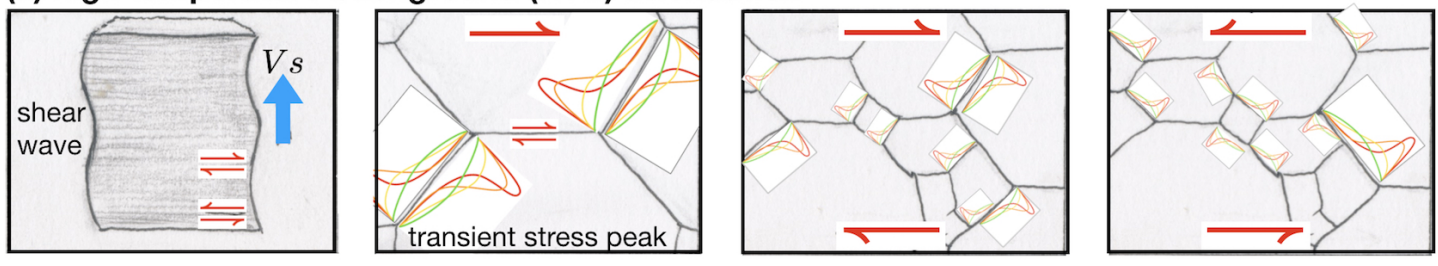

(b) microstructural effects on HTB attenuation
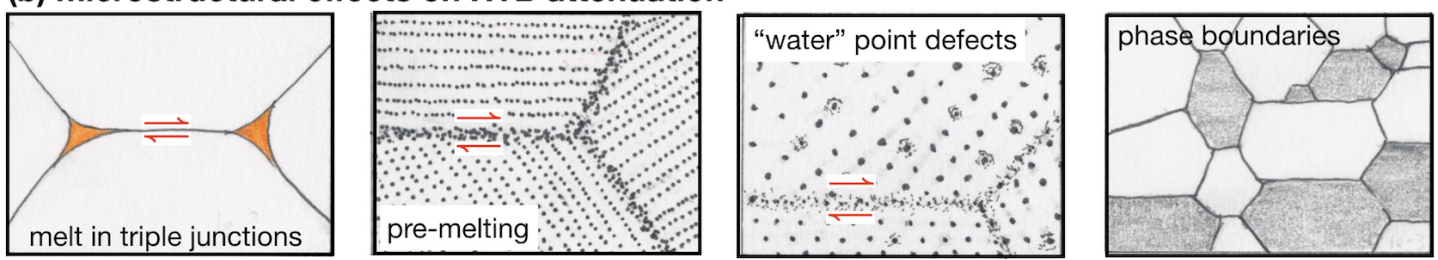

(c) secondary dissipative mechanisms
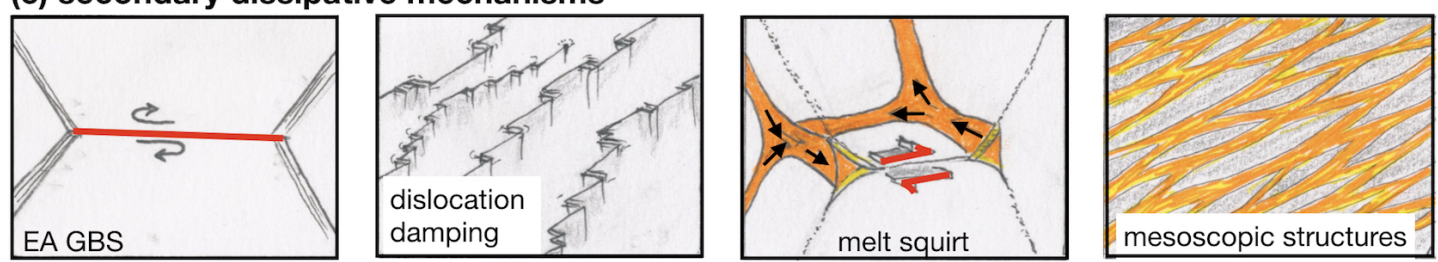

Figure 1: Transient creep/attenuation mechanisms (a) High temperature background (HTB). (1) a propagating shear wave will have energy attenuated at the grain scale (2) at high temperature by transient diffusion creep, that occurs when sliding on a grain boundary causes peaks in traction at grain corners (red lines) that drive rapid local diffusion and diminish as the diffusion relaxes stress towards the traction profile of steady state creep (green lines). $(3,4)$ As the wave arrives and passes, tractions develop on one set of grain edges and then switch; the total dissipation depends on frequency. (b) Properties that affect the HTB: (1) melt tubules (with topology determined by surface tension) at grain triple junctions can aid in rapid diffusion. (2) premelting- or sub-solidus disordering of the grain boundary- leads to increased diffusivity which also relaxes traction peaks more quickly, as can (3) water-related defects in crystals or on grain boundaries and (4) phase boundaries. (c) secondary dissipative mechanisms include (1) elastically accommodated grain boundary sliding (eaGBS), (2) dislocation damping, (3) melt squirt, and (4) potential meso-scopic structures. 

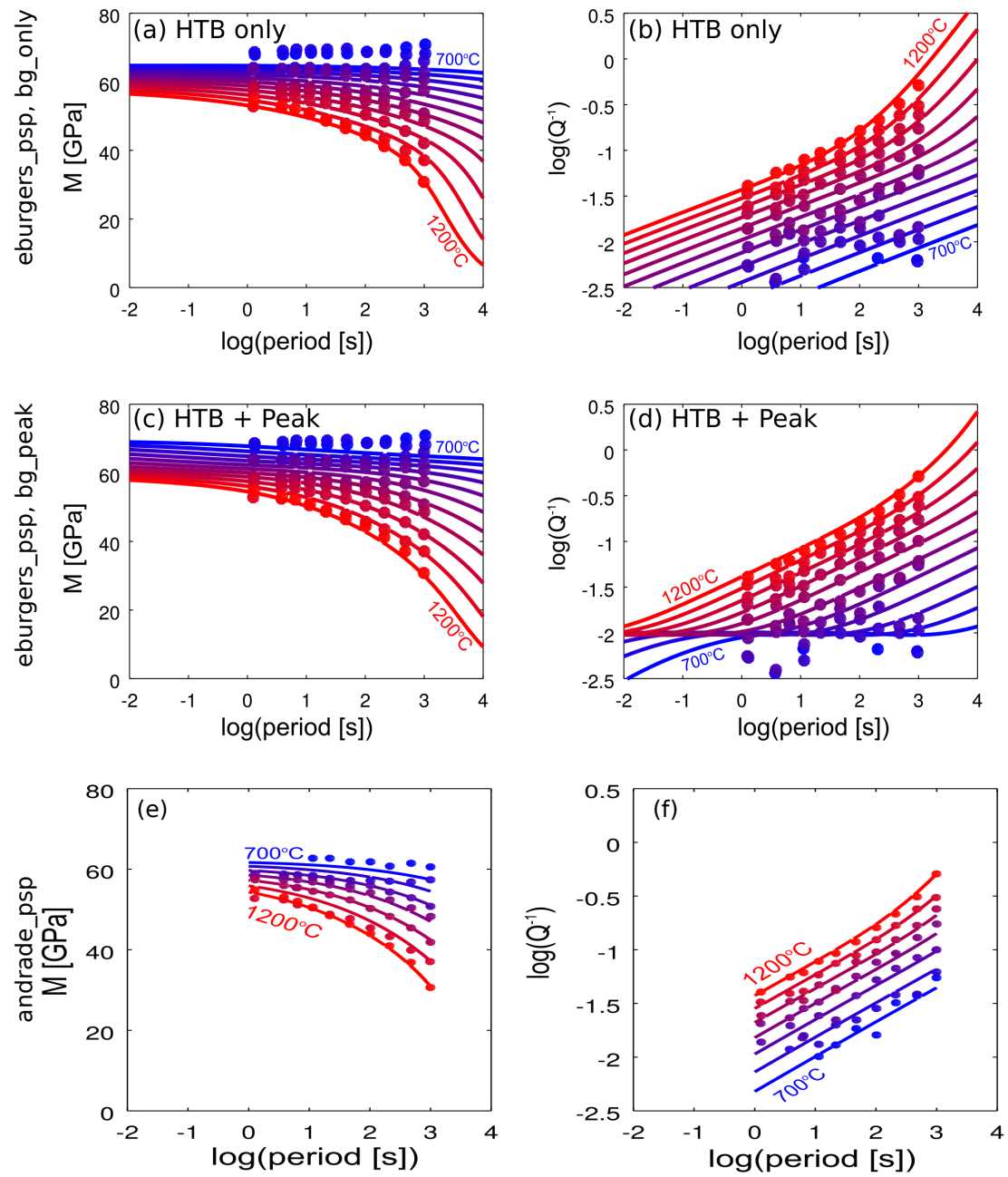

Figure 2: Fits to attenuation data from Jackson and Faul (2010), to benchmark constitutive model modules in the VBRc: extended Burgers (eburgers_psp) and Andrade (andrade_psp) models scaled by the pseudoperiod method. Data corresponds to the single-sample fit in figure 1 of Jackson and Faul (2010). 

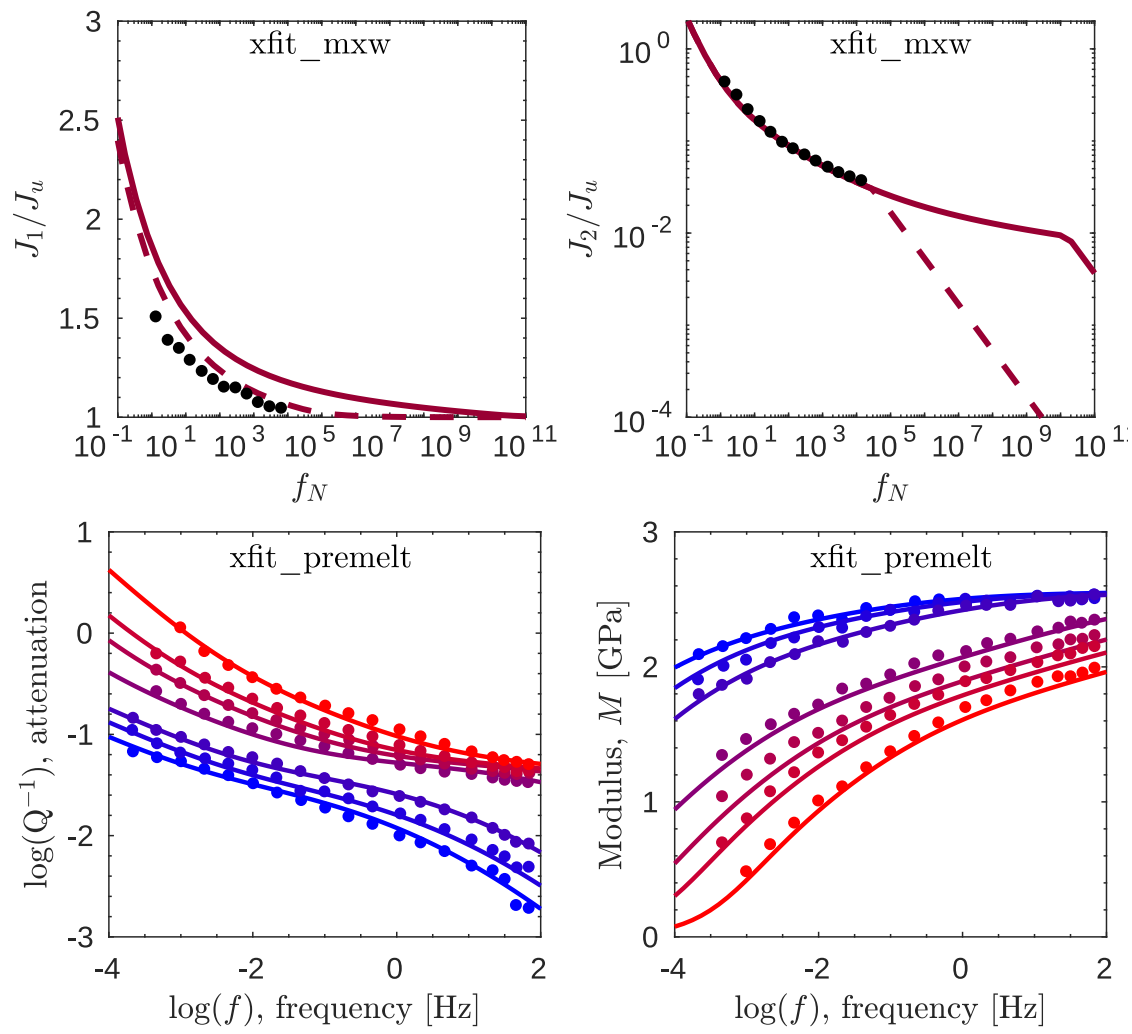

Figure 3: Fits to attenuation data to benchmark our constitutive model modules in the VBRc: empirical relaxation spectrum fits (xfit), for Maxwell (top, xfit_mxw) and pre-melting (bottom, xfit_premelt) scaling methods. The xfit_mxw panels show the dependency of (a) normalized storage compliance $J_{1} / J_{u}$ where $J_{u}$ is the unrelaxed compliance and (b) normalized loss compliances, $J_{1} / J_{u}$ on Maxwell-normalized frequency, $f_{N}$. The solid and dashed curves correspond to the two fits of the relaxation spectrum calculated by McCarthy and Takei (2011) and the xfit_mxw data are from figures 14 and 15 of McCarthy and Takei (2011). The xfit_premelt panels show the frequency dependence of attenuation $Q^{-1}$ and (d) modulus $M$ with data from figure 10 and table 4 of Yamauchi and Takei (2016). 

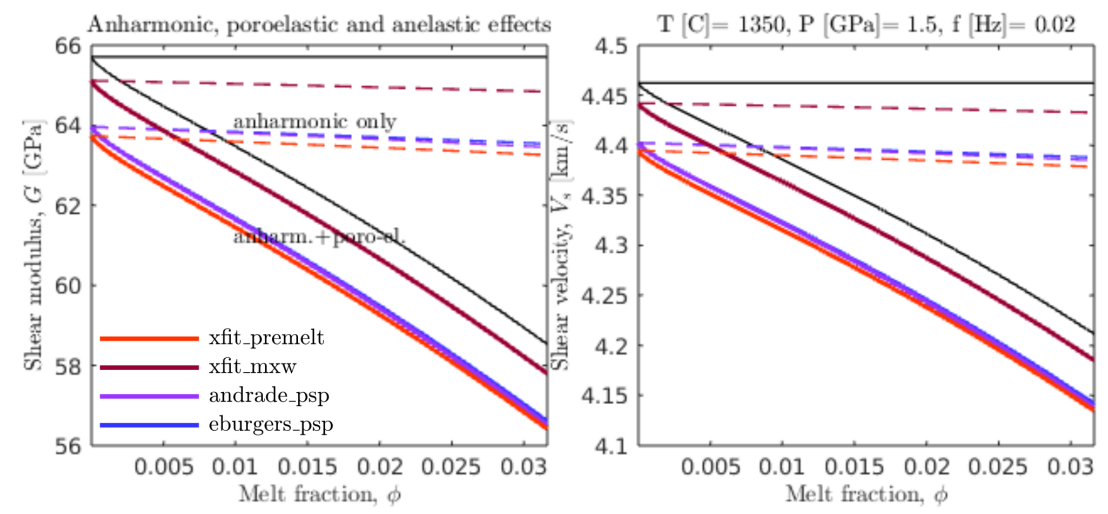

Figure 4: Influence of poro-elasticity on (a) Shear modulus, G. Thin black lines show the reference modulus and the poroelastic effect; colored dashed lines show anelastic effects imposed on the unrelaxed modulus with no poroelastic effect, and solid lines show anelastic effects imposed on the modulus with the poroelastic effect. (b) corresponding $V s$ values (not including effects of melt on the density). 


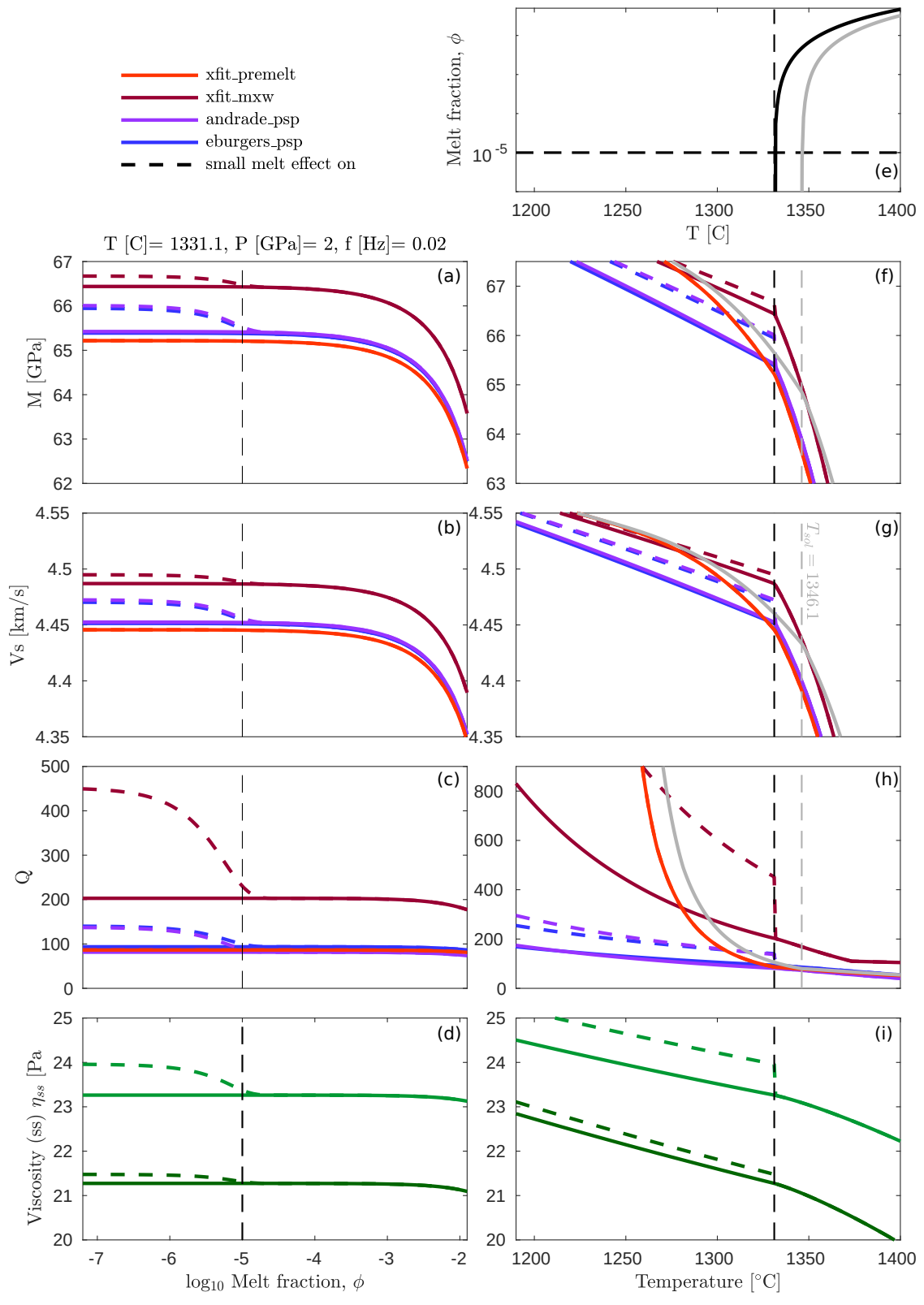

Figure 5: Melt effects for the different anelastic methods. Left column (a)-(d) is at fixed temperature and variable melt fraction. Dashed lines show the parameterized correction for accounting for small melt fractions from Holtzman (2016), see Section 2.2. Right column (e)-(i) uses an equillibrium batch melting approximation to calculate melt fraction as a function of temperature. In $\mathbf{g , h}, \mathbf{h}$ the grey lines show the effect of changing the solidus temperature on the Xfit_premelt method, leaving all other parameters constant. Plotted variables are as follows: shear modulus $G$, shear wave velocity $V s$, quality factor $Q$, steady state viscosity $\eta_{s s}$, light green= diffusion creep, dark green $=$ composite viscosity . 

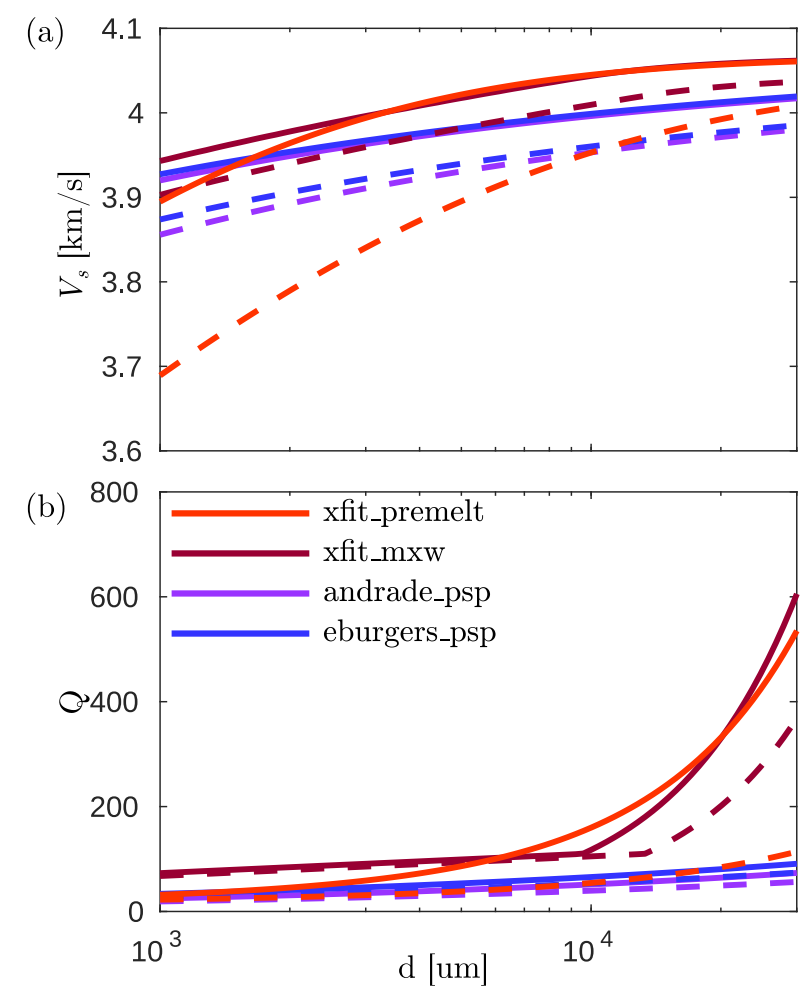

Figure 6: Grain size dependence of (a) shear wave velocity $V_{s}$ and (b) quality factor $Q$ for the four anelastic methods at a temperature below (solid curves) and above (dashed curves) the solidus from Fig. 4e-4i. The lower temperature is at $1300^{\circ} \mathrm{C}$ and the upper at $1350^{\circ} \mathrm{C}$, all other state variables are the same as in Fig. 4e-4i. 
Vs, $Q$ as a function of T, $\phi$, d (2 GPa, $50-150 \mathrm{~s})$
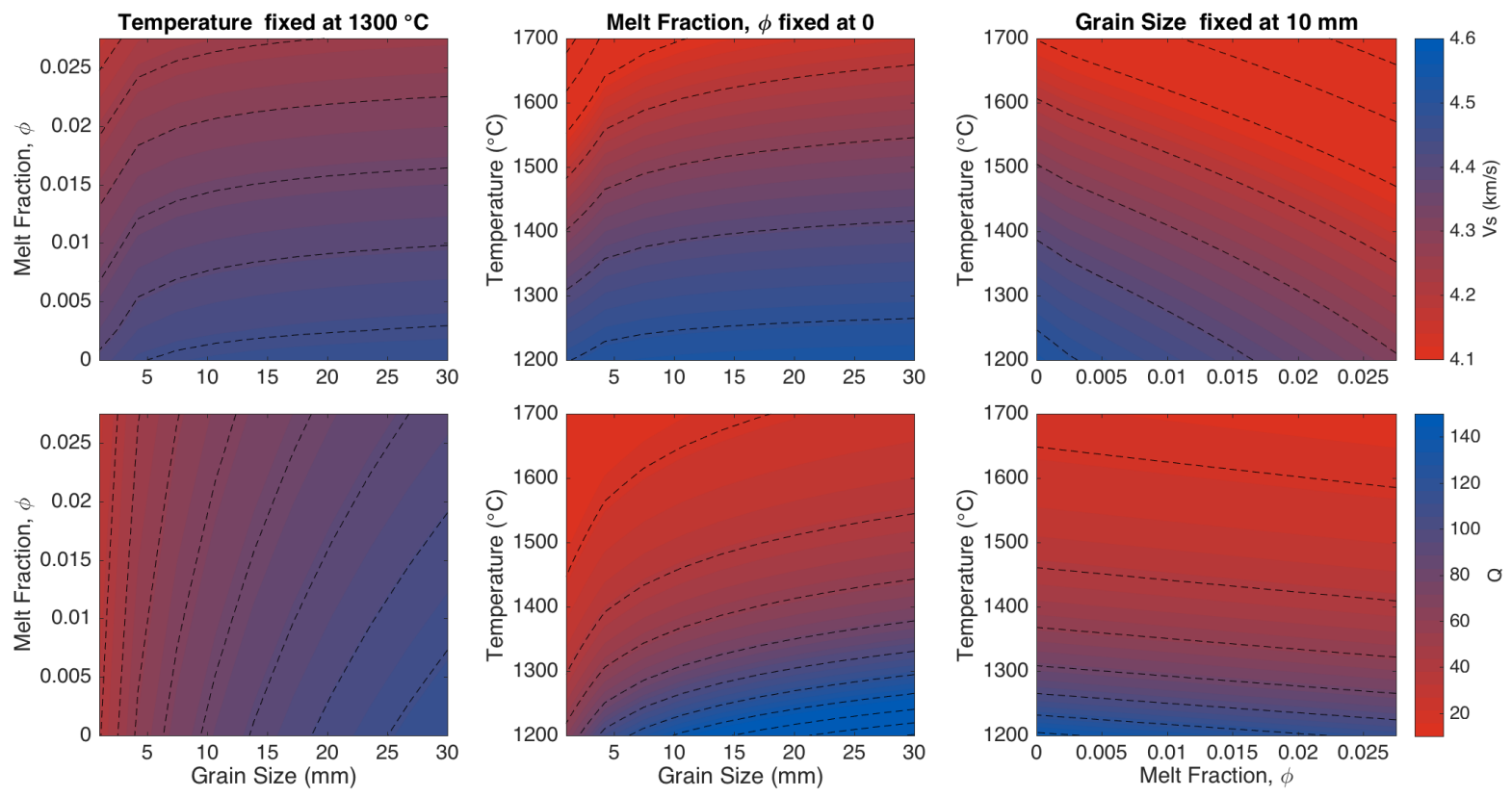

Figure 7: Look-up Table (LUT) slices showing tradeoffs between temperature, grain size, and melt fraction at a fixed pressure and frequency band for shear wave velocity $V_{s}$ (top row) and quality factor $Q$ (bottom row). These calculations use the andrade_psp anelastic method. 


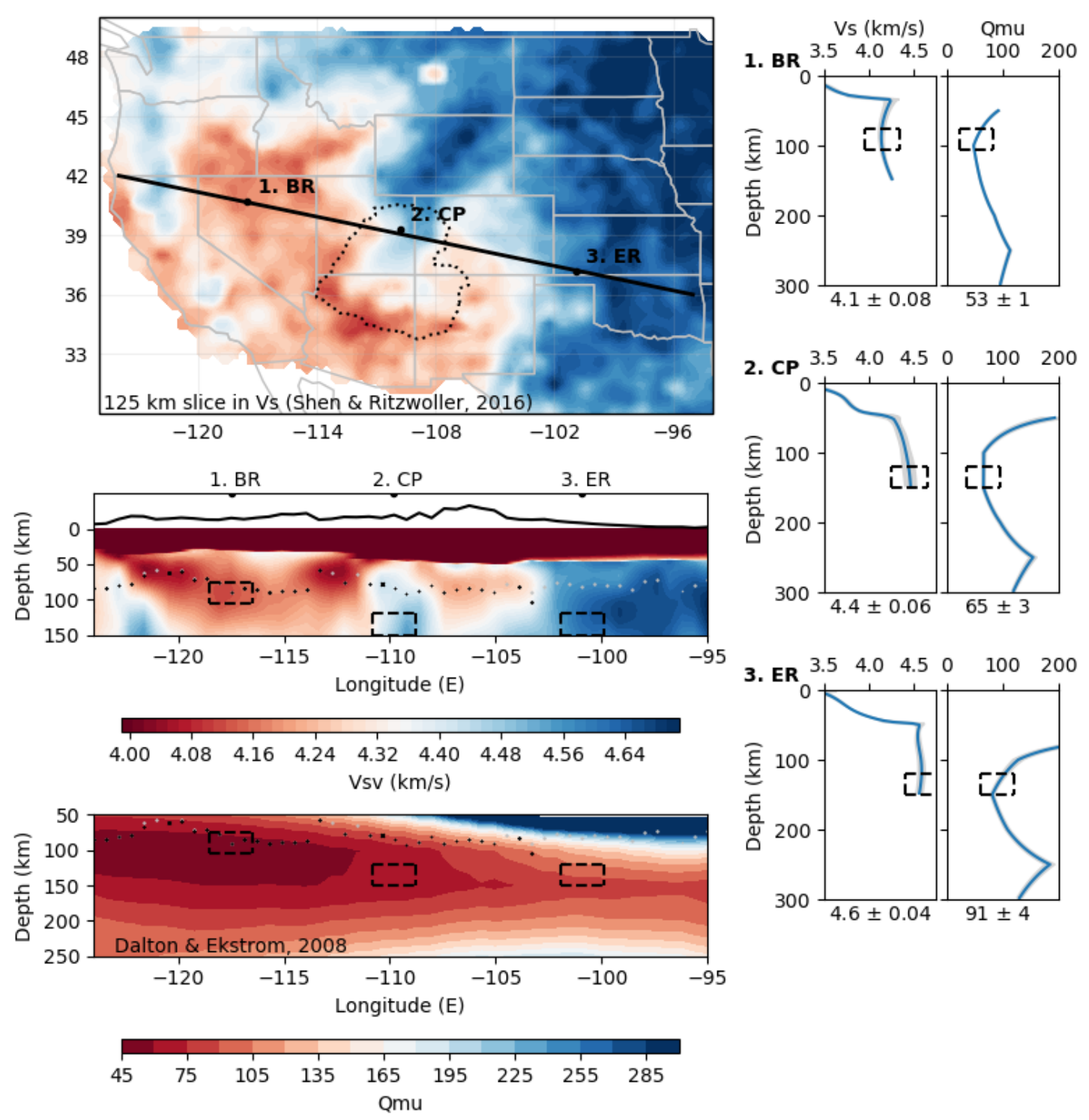

Figure 8: The representative sites and corresponding data used in the Bayesian inference. Sites are 1. the Basin and Range (BR), 2. the Colorado Plateau interior (CP) and 3. the cratonic interior east of the Rio Grande (ER). The regional map and middle cross-section shows shear wave velocity $V_{s}$ from Shen and Ritzwoller (2016), the bottom cross-section shows $Q$ from Dalton et al. (2008). The profiles to the right show $V_{s}$ and $Q$ vs. depth for each site. The dashed black boxes show the depth range used to calculate single values of $V_{s}$ and $Q$ used in the Bayesian Inference. 

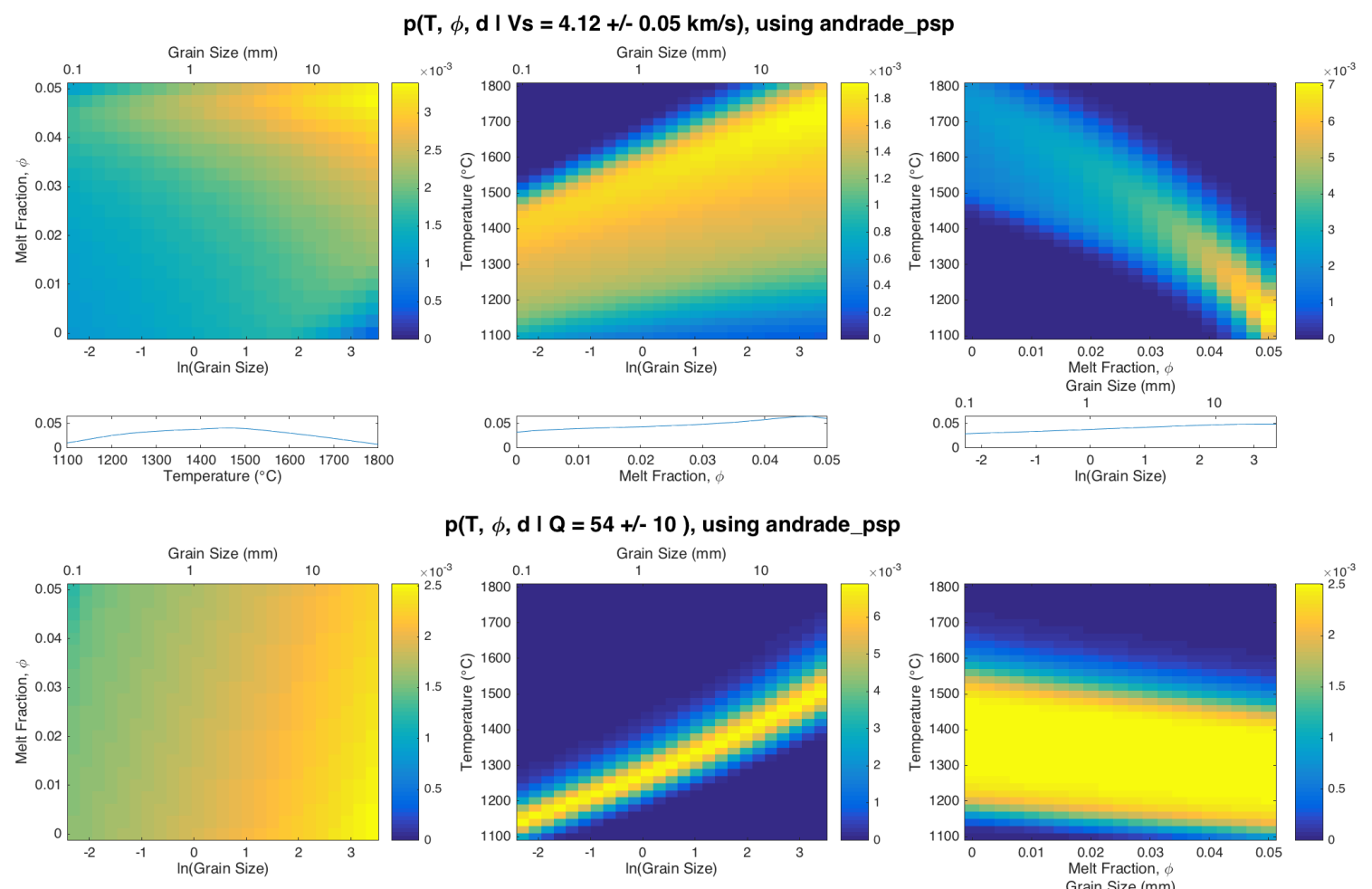

$\mathrm{p}(\mathrm{T}, \phi, \mathrm{d}$ I Q $=\mathbf{5 4}+/-10)$, using andrade_psp
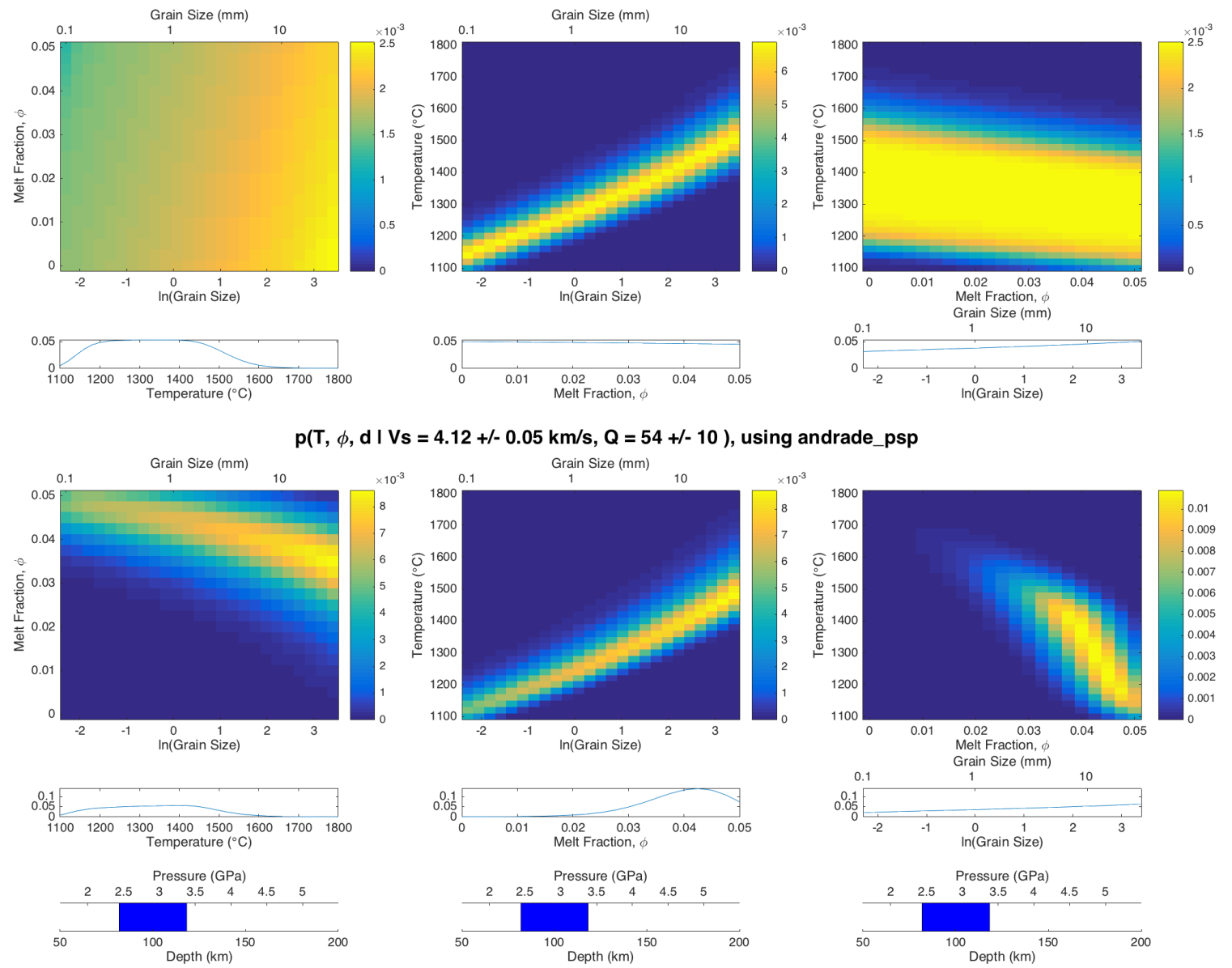

Figure 9: Probability distributions of melt fraction $\phi$, temperature $T$ and grain/subgrain size $d$ for the Basin and Range using the Andrade pseudoperiod scaling (andrade_psp) for three cases: (top row) single parameter inference using shear wave velocity $V_{s}: p\left(\phi, T, d \mid V_{s}\right)$, (middle row) single parameter inteference using quality factor $Q p(\phi, T, d \mid Q)$, and (bottom row) joint inference using $V_{s}$ and $Q: p\left(\phi, T, d \mid V_{s}, Q\right)$. The 2D plots are the probability summed over the third variable that is not plotted and the 1D plots are the marginal probability of the that thrid variable. The bottom pressure-depth plots show the pressure range of the observation. 


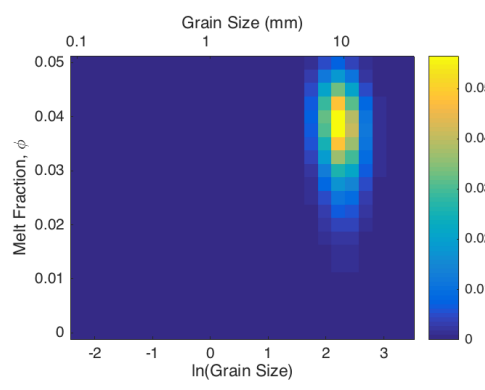

$1 \mathrm{~cm}$ median grain/subgrain size
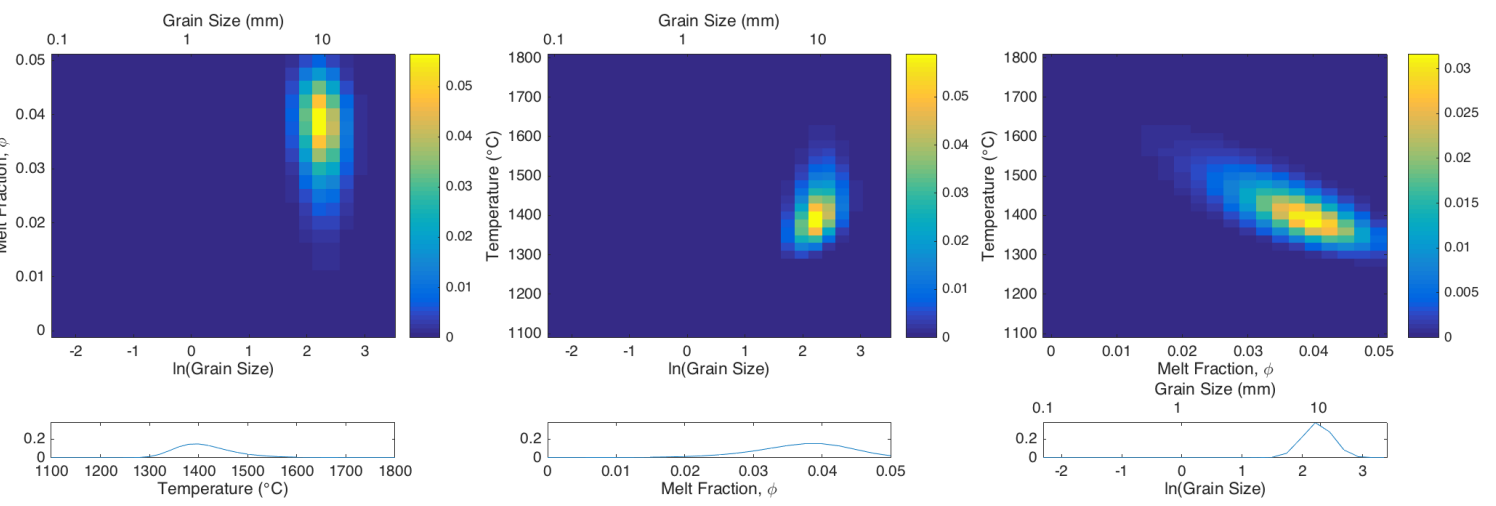

$1 \mathrm{~mm}$ median grain/subgrain size
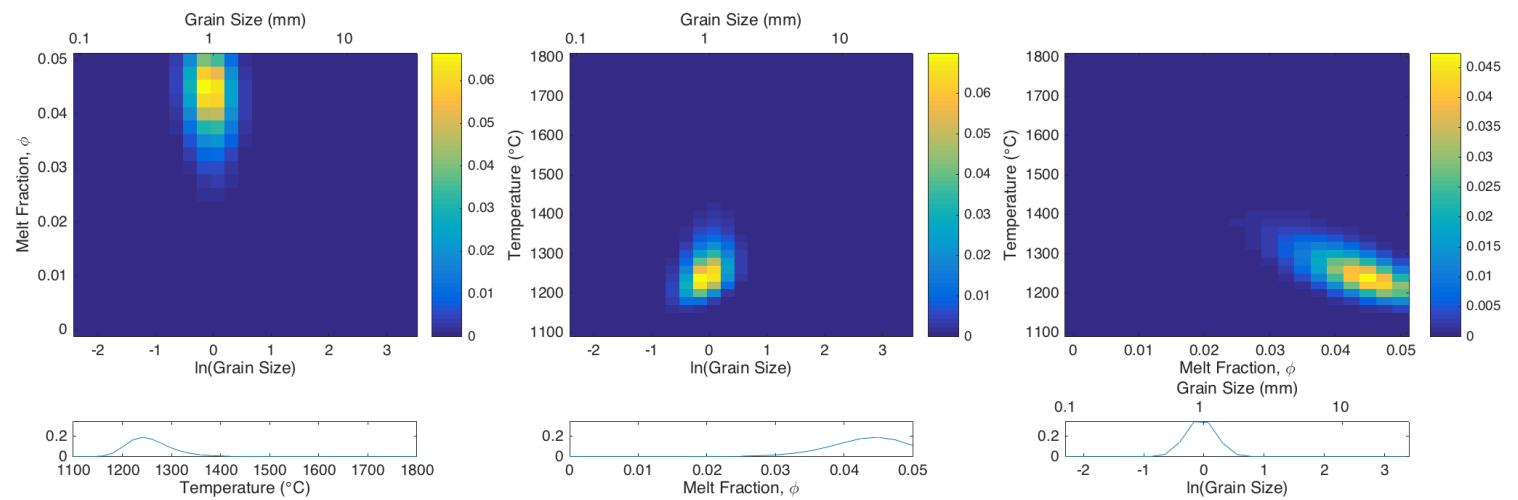

Figure 10: Joint probability distribution $p\left(\phi, T, d \mid V_{s}, Q\right)$ for the Basin and Range using the Andrade pseudoperiod scaling and assuming a log-normal distribution for the prior model of grain/subgrain size with median grain/subgrain size of (top row) $1 \mathrm{~cm}$ and (bottom row) $1 \mathrm{~mm}$. See figure 9 for an explanation of the 2D and 1D plots. 

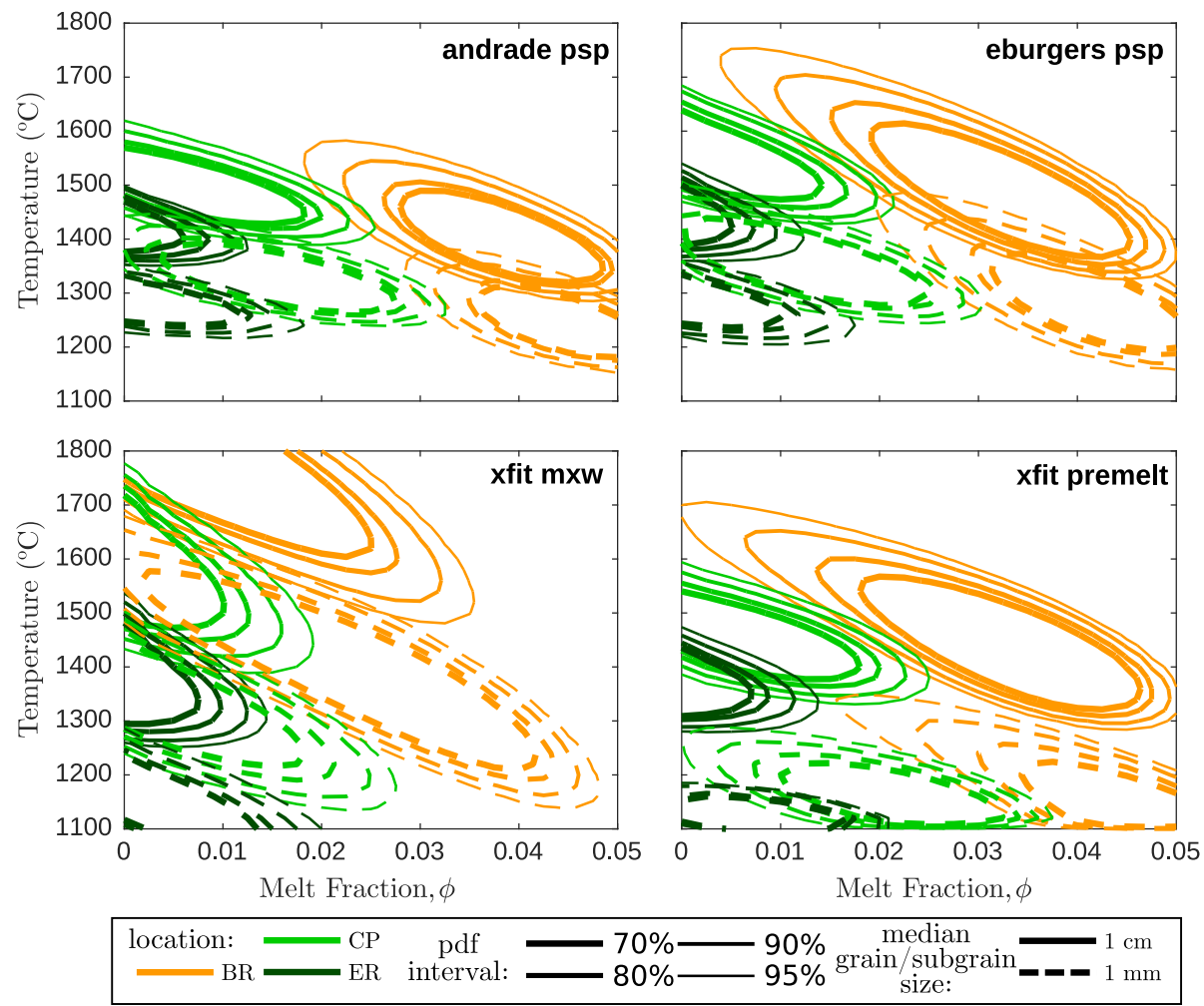

Figure 11: Likely melt fraction - temperature fields for each anelastic method and location for the two prior model cases in figure 10. Line color corresponds to location (abbreviations defined in Fig. 8), line thickness corresponds to the probability distribution interval (e.g., the thickest lines contain $70 \%$ of the distribution), and line style corresponds to the median grain/subgrain size of the prior model. 

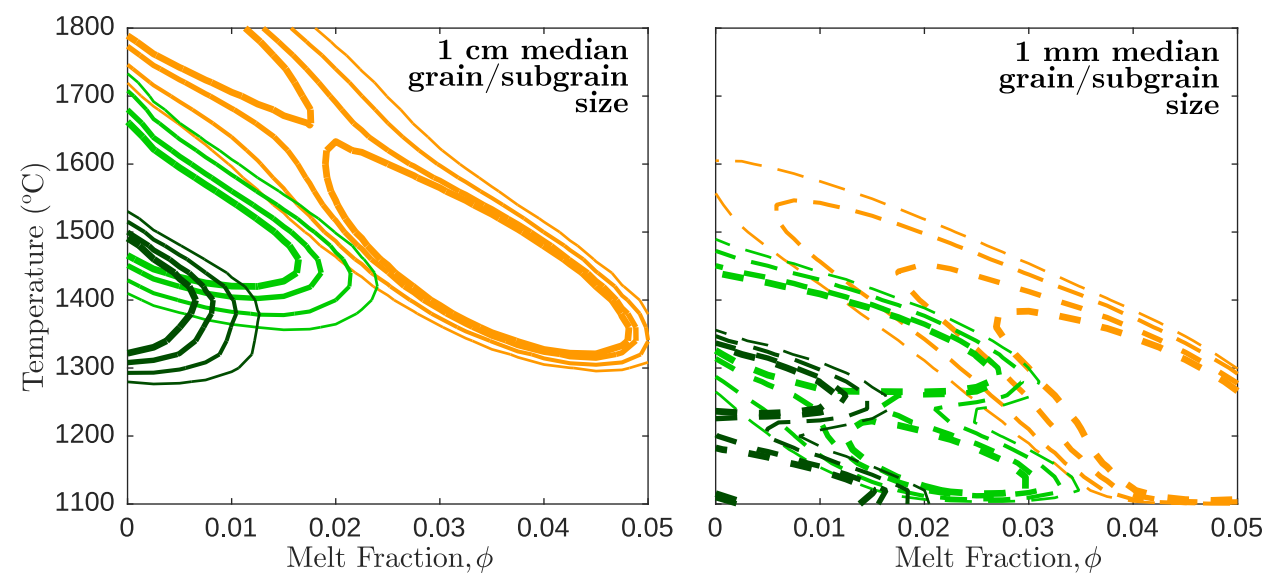

Figure 12: Likely melt fraction - temperature fields for each location using an ensemble weighting of the probability distributions for each anelastic method. See the legend and caption of figure 11 for description of line colors and styles. 